\title{
Nonlocal quantum field theory without acausality and nonunitarity at quantum level: is SUSY the key?
}

\author{
Andrea Addazi ${ }^{1,2}$ and Giampiero Esposito ${ }^{3}$ \\ ${ }^{1}$ Dipartimento di Fisica, Università di L'Aquila, 67010 Coppito AQ, Italy \\ ${ }^{2}$ Laboratori Nazionali del Gran Sasso (INFN), 67010 Assergi AQ, Italy \\ ${ }^{3}$ Istituto Nazionale di Fisica Nucleare, Sezione di Napoli, \\ Complesso Universitario di Monte S. Angelo, \\ Via Cintia Edificio 6, 80126, Napoli, Italy
}

(Dated: March 16, 2015)

\begin{abstract}
The realization of a nonlocal quantum field theory without losing unitarity, gauge invariance and causality is investigated. It is commonly retained that such a formulation is possible at tree level, but at quantum level acausality is expected to reappear at one loop. We suggest the the problem of acausality is, in a broad sense, similar to the one about anomalies in quantum field theory. By virtue of this analogy, we suggest that acausal diagrams resulting from the fermionic sector and the bosonic one might cancel each other, with a suitable content of fields and suitable symmetries. As a simple example, we show how supersymmetry can alleviate this problem in a simple and elegant way, i.e., by leading to exact cancellations of harmful diagrams, to all orders of perturbation theory. An infinite number of divergent diagrams cancel each other by virtue of the nonrenormalization theorem of supersymmetry. However, supersymmetry is not enough to protect a theory from all acausal divergences. For instance, acausal contributions to supersymmetric corrections to $D$-terms are not protected by supersymmetry. On the other hand, we show in detail how supersymmetry also helps in dealing with $D$-terms: divergences are not cancelled but they become softer than in the nonsupersymmetric case. The supergraphs' formalism turns out to be a powerful tool to reduce the complexity of perturbative calculations.

PACS numbers: $03.70 .+\mathrm{k}, 11.25 . \mathrm{w}, 11.30 . \mathrm{Pb}, 12.60 . \mathrm{Jv}$
\end{abstract}

Keywords: Quantum field theory, supersymmetry, string theory 


\section{INTRODUCTION}

The successes of local quantum field theories are well known. They make it possible to understand the current results in particle physics. The first famous success of a local quantum field theory was the capability to account for the anomaly in the magnetic moment of the electron, with respect to the prediction of Dirac's equation, calculated independently by Tomonaga [1], Schwinger [2, 3] and Feynman [4 6]. However, one-loop calculations like these lead to divergences. These are originating from locality: we are assuming, in a local quantum field theory, that quantum fields are interacting at a vertex point in spacetime. On the other hand, we can control these divergences through renormalization techniques, with the subtraction of counterterms. Among physicists, the most famous skeptical about this practice was Dirac himself [7]. He viewed renormalization as a trick without mathematical consistency. On the other hand, renormalization has a well understood physical meaning in the interpretation of quantum field theories as effective field theory, formalized through the renormalization group approach. In fact the infinities are cutoff by a scale $\Lambda$, possibly coincident with the Planck scale of quantum gravity or so, where an unknown new physics, supposed completely different from local quantum field theories, has to be considered. This was inspired by condensed matter physics. In fact, in a material, it is straightforward to understand why a a physical short-distance regulator has to be considered, because of the transition from the continuum to the atomic discretization. In this sense, the Dirac doubts were premature for his times. However, the various different approaches to quantum gravity like string theory [8], loop quantum gravity [9], noncommutative geometry [10] seem to lead again to the same problem: nonlocality in a quantum field theory. In fact, string [11] theory $^{1}$ is intrinsically nonlocal, and the presence of Kaluza-Klein towers of infinitely many higher-spin fields is a general feature, leading to nonlocalities ${ }^{2}$. For example, we remember

${ }^{1}$ For string-inspired phenomenology see Ref. 12,15 . Exotic stringy instantons can indeed induce nonperturbative couplings leading to new physical effects, for example in ultra-cold neutron physics. These effects are computable and perfectly controllable in a large class of string models, leading to effects that cannot be generated at all in gauge theories.

${ }^{2}$ Nonlocal classical infrared extensions of General Relativity were also proposed in order to simulate, dynamically, the apparent presence of dark energy in our Universe [16]. See also [17, 18] for recent developments in this subject. Similarly, Lorentz-violating infrared extensions of the Einstein-Hilbert action might be another viable alternative to nonlocal gravity. For instance, a Lorentz violating mass term for the graviton can be added to the Einstein-Hilbert action without the introduction of ghosts 19 21]. However, other 
the case of a spin $s=3$ field, with nonlocal equation of motion: a local quantum field theory approach does not permit to quantize such a theory. On the other hand, in loop quantum gravity [9], the "discretization" of spacetime itself naturally leads to the question about the presence of quantum fields of matter in such a spacetime. In noncommutative geometry [10], the peculiar topology is inducing new nonlocal interaction terms in the functional integral of matter fields. This could suggest that the next step of the road to quantum gravity might be to solve the question about the admissibility of a nonlocal quantum field theory. A lot of attempts to formulate a quantum field theory without locality have been made, in order to evade the problem of ultraviolet divergencies in a consistent way. The idea is to circumvent the ultraviolet infinities by replacing local vertices with nonlocal, smeared ones. The first suggestion was made by Wataghin in Ref. [23]. However, it was realized that a lot of problems in a nonlocal quantum field theory have to be solved in order to have a meaningful and computable theory (for example, see the review in Ref. [24]). The main difficulty is to construct a theory preserving unitarity, gauge invariance and causality at the same time [25 30]. Unitarity of the S-matrix is a necessary condition for a "meaningful theory" that evaluates probabilities. For example, this condition automatically saves the theory from negative-norm states on-shell. On the other hand, nonlocal interactions have to be compatible with a local gauge symmetry: nonlocalization of the vertices has to be compatible with the essential consequences of a local gauge symmetry. Eventually, causality just requires that the commutator between two fields, located at two different spacetime positions, has to vanish for a spacelike distance between the two spacetime locations. A consistent formulation of a nonlocal quantum field theory preserving unitarity, local gauge symmetry and causality at tree level was presented in Refs. [26 30] as a $\lambda \phi^{4}$ model, simply generalizable to a Yang-Mills model in the same paper just cited above. These models are realized through the introduction of auxiliary fields, not existing on-shell but to be considered off-shell (at least with exception of few particular cases of gauge choices). Unfortunately, as shown in Refs. [31, 32], such models are losing causality just at one loop. In particular, in the simplest case of a massless self-interacting model $\lambda \phi^{4}$, one-loop diagrams with propagating auxiliary fields lead to divergences: in a scattering process $\phi \phi \rightarrow \phi \phi$, the divergence occurs

phenomenological difficulties, such as geodetic instabilities around stars and black holes for large part of the parameters' space, reject a large class of these extensions [22]. 
at lower order $\mathcal{S} \sim s^{2}+t^{2}+u^{2}$, where $\mathcal{S}$ is the $S$-matrix describing such a scattering.

Is it possible to avoid this causality violation at quantum level? This is the issue that we are considering in this paper. In fact, we would like to suggest a possible analogy with the problem of the anomalies in quantum field theory. Anomalies are in general the loss of a gauge symmetry of a classical action, when it is considered at quantum level. For instance, in the standard model, we have an anomalous contribution from triangle diagrams, but all contributions resulting from quarks and leptons cancel each other, leading to a consistent theory. We would like to stress that the electroweak theory $S U(2) \times U(1)$ proposed by Glashow, Weinberg, Salam would be inconsistent because of local anomalies if we were not considering the counter contributions from the quark sector - in this sense, the electroweak theory naturally suggests its own gauge-group extension. Thus, our attitude about acausalities could be the same: we have to construct a nonlocal quantum field theory with a field content leading to automatic cancellations of harmful quantum diagrams.

In this paper, we are suggesting that a supersymmetric nonlocal quantum field theory is automatically free of causality violations resulting from supersymmetric F-terms to all orders of perturbation theory. This is a direct consequence of the nonrenormalization theorem of supersymmetry [33, 34]. If F-terms cannot be corrected by radiative contributions, automatically we will obtain a cancellation of all acausal $n$-loop contributions. In particular, we consider a susy generalization of the Eliezer-Woodard-Moffat-Kleppe-Evans model, described in Refs. [26 30] for a $\lambda \phi^{4}$ model and a gauge theory. We also stress that our result is valid only under the particular prescription described in Refs. [26 30] ${ }^{3}$. Curiously, a supersymmetric generalization of EWMKE was not considered before in literature. We would like to clarify that our model cannot be considered in a Lorentz violating space-time and non-commutative geometries. In particular, we will consider a nonlocal Wess-Zumino and a nonlocal super Yang-Mills, showing how F-terms remain causal forever. Unfortunately, acausalities will reappear by means of $D$-terms in a nonlocal super Yang-Mills: the nonrenor-

\footnotetext{
${ }^{3}$ An alternative approach to the one considered in this paper was studied in [35 38], with several implications in cosmology and LHC. In this case, issues about gauge invariance and unitarity are not considered as a problem: an effective string-inspired approach is developed. Recently, questions about string theory and causality were considered in Refs. [39] and [40]. In particular, the scattering of gravitons in Regge limit was considered and the entire Kaluza-Klein tower appears to cure acausal contributions [40]. Questions about causality at all orders of perturbations in string theory remain open. For useful discussions about String amplitudes and possible LHC implications see also Refs. [41, 42].
} 
malization theorem does not protect the theory against these corrections. For this reason, we suggest that only an infinite number of bosons and fermions can cancel all the infinite number of dangerous divergences. In this sense, supersymmetry is a powerful framework in order to cancel an infinite number of harmful diagrams, but unfortunately it is not enough to cancel all the acausalities. However, we show that supersymmetry also gets softer acausal violations with respect to the nonsupersymmetric case. For these reasons, supersymmetry seems to be the key for the realization of a nonlocal quantum field theory.

In Sec. II, we build the nonlocal Wess-Zumino model. In Sec. III, we discuss the main points about the consistency of this model, considering gauge transformations, unitarity, causality. In Secs. IV-V-VI, we get the main result of the paper: the cancellations of harmful acausal loops resulting from $F$-terms. In Sec. VII, we discuss the generalizations of our results about Wess-Zumino models by considering a nonlocal super Yang-Mills model, discussing also how in this case the cancellations occur for F-terms. In Sec. VIII, we are ending with our comments and perspectives. Thus, for the self-consistency of our paper, we find it necessary to summarize the basic properties of local Wess-Zumino [43] and local super Yang-Mills models, nonlocal nonsupersymmetric $\lambda \phi^{4}$ model in Appendices, in order to facilitate the logical step towards their nonlocal generalization.

\section{NONLOCAL WESS-ZUMINO MODEL}

In this section, we are generalizing the local Wess-Zumino model ( $\mathcal{N}=1$ susy) in (B8) $)$ (B9), to a nonlocal one. We are introducing an extra chiral supermultiplet $\Phi^{2}=\left\{F^{2}, \phi^{2}, \psi^{2}\right\}$ playing the role of auxiliary superfield, in order to construct a nonlocal model, without losing rigid supersymmetry. In other words, we are extending a nonlocal scalar model invariant under the global Poincaré group to a nonlocal chiral superfield model invariant under the global superPoincaré group.

We consider the following auxiliary supersymmetric action ${ }^{4}$ :

$$
S\left[\hat{F}^{1}, F^{2}, \hat{\phi}^{1}, \phi^{2}, \hat{\psi}^{1}, \psi^{2}\right]=\mathcal{F}\left[\hat{F}^{1}, \hat{\phi}^{1}, \hat{\psi}^{1}\right]-\mathcal{A}\left[F^{2}, \phi^{2}, \psi^{2}\right]+\mathcal{I}\left[\hat{F}^{1}+F^{2}, \hat{\phi}^{1}+\phi^{2}, \hat{\psi}^{1}+\psi^{2}\right]
$$

\footnotetext{
${ }^{4}$ We are assuming for the moment a number of spacetime dimensions $D=4$, but this formalism can be extended to an arbitrary number of spacetime dimensions. This framework might be of interest for string theories.
} 
with

$$
\begin{gathered}
\mathcal{F}\left[\hat{F}^{1}, \hat{\phi}^{1}, \hat{\psi}^{1}\right]=\int \mathrm{d}^{4} x\left[\hat{F}_{i}^{1^{\dagger}} a_{i j}^{1} \hat{F}_{j}^{1}+\hat{\phi}_{i}^{1^{\dagger}} b_{i j}^{1} \hat{\phi}_{j}^{1}+\overline{\hat{\psi}}_{i}^{1^{\dagger}} f_{i j}^{1} \hat{\psi}_{j}^{1}\right], \\
\mathcal{A}\left[F^{2}, \phi^{2}, \psi^{2}\right]=\int \mathrm{d}^{4} x\left[F_{i}^{2^{\dagger}} a_{i j}^{2} F_{j}^{2}+\phi_{i}^{2^{\dagger}}(x) b_{i j}^{2} \phi_{j}^{2}(x)+\bar{\psi}_{i}^{2^{\dagger}} f_{i j}^{2} \psi_{j}^{2}\right],
\end{gathered}
$$

where $\hat{\phi}^{1}, \hat{\psi}^{1}, \hat{F}^{1}$ are the "smeared fields". For simplicity, we might choose an exponential smearing operator for the scalar field defined as

$$
\hat{\phi}_{1} \equiv \mathcal{E}^{-1} \phi_{1}, \quad \mathcal{E} \equiv \mathrm{e}^{b^{1} / 2 \Lambda^{2}}
$$

Automatically, in order to preserve the (rigid) supersymmetry transformations

$$
\begin{gathered}
\delta_{\zeta} \phi_{i}^{1,2}=\zeta \psi_{i}^{1,2} \\
\delta_{\zeta} \psi_{i}^{1,2}=-\mathrm{i} \sigma^{\mu} \mathrm{i} \sigma_{2} \zeta^{*} \partial_{\mu} \phi_{i}^{1,2}+\zeta F_{i}^{1,2} \\
\delta_{\zeta} F_{i}^{1,2}=-\mathrm{i} \zeta^{*} \bar{\sigma}^{\mu} \partial_{\mu} \psi_{i}^{1,2}
\end{gathered}
$$

we have to take rigidly the same smearing prescription for the superpartners, i.e.

$$
\hat{\psi}^{1} \equiv \mathcal{E}^{-1} \psi_{1}, \quad \hat{F}^{1} \equiv \mathcal{E}^{-1} F^{1}
$$

In (2.1) we also define the following prescriptions for auxiliary fields ( $I$ being the identity operator)

$$
a^{2} \equiv \frac{a^{1}}{\left(\mathcal{E}^{2}-I\right)}, \quad b^{2} \equiv \frac{b^{1}}{\left(\mathcal{E}^{2}-I\right)}, \quad f^{2} \equiv \frac{f^{1}}{\left(\mathcal{E}^{2}-I\right)}
$$

The action of the nonlocal theory is obtained as

$$
\hat{S}\left[\hat{F}^{1}, \hat{\phi}^{1}, \hat{\psi}^{1}\right]=S\left[\hat{F}^{1}, \hat{\phi}^{1}, \hat{\psi}^{1}, F^{2}\left[\hat{F}^{1}, \hat{\phi}^{1}, \hat{\psi}^{1}\right], \phi^{2}\left[\hat{F}^{1}, \hat{\phi}^{1}, \hat{\psi}^{1}\right], \psi^{2}\left[\hat{F}^{1}, \hat{\phi}^{1}, \hat{\psi}^{1}\right]\right]
$$

where $F^{2}\left[\hat{F}^{1}, \hat{\phi}^{1}, \hat{\psi}^{1}\right], \phi^{2}\left[\hat{F}^{1}, \hat{\phi}^{1}, \hat{\psi}^{1}\right], \psi^{2}\left[\hat{F}^{1}, \hat{\phi}^{1}, \hat{\psi}^{1}\right]$ solve the classical equations

$$
\frac{\delta S}{\delta \phi^{2}}=\frac{\delta S}{\delta \psi^{2}}=\frac{\delta S}{\delta F^{2}}=0
$$

Of course, we can also rewrite the auxiliary action (2.1) in a form explicitly invariant under (rigid) supersymmetry:

$$
\int \mathrm{d}^{4} x\left[\mathcal{G}\left\{\int \mathrm{d}^{2} \theta \mathrm{d}^{2} \bar{\theta}\left(\mathcal{K}^{1}\left(\bar{\Phi}^{1}, \Phi^{1}\right)+\mathcal{K}^{2}\left(\bar{\Phi}^{2}, \Phi^{2}\right)\right)\right\}+\int \mathrm{d}^{2} \theta \mathcal{W}\left(\Phi^{1}+\Phi^{2}\right)+\int \mathrm{d}^{2} \bar{\theta} \overline{\mathcal{W}}\left(\bar{\Phi}^{1}+\bar{\Phi}^{2}\right)\right]
$$


where $\mathcal{K}^{1,2}$ and $\mathcal{G}\{\mathcal{O}\}$ are encoding the nonlocal bilinear terms, for the standard and the auxiliary chiral superfields ${ }^{5}$. Let us recall that

$$
\Phi=\phi+\sqrt{2} \theta \psi(x)+\mathrm{i} \theta \sigma^{\mu} \bar{\theta} \partial_{\mu} \phi(x)-\theta \theta F(x)-\frac{\mathrm{i}}{\sqrt{2}} \theta \theta \partial_{\mu} \psi(x) \sigma^{\mu} \bar{\theta}-\frac{1}{4} \theta \theta \bar{\theta} \bar{\theta} \square \phi(x),
$$

and

$$
\bar{\Phi}=\bar{\phi}+\sqrt{2} \bar{\theta} \bar{\psi}(x)+\mathrm{i} \bar{\theta} \sigma^{\mu} \theta \partial_{\mu} \bar{\phi}(x)-\bar{\theta} \bar{\theta} F(x)-\frac{\mathrm{i}}{\sqrt{2}} \bar{\theta} \bar{\theta} \partial_{\mu} \bar{\psi}(x) \sigma^{\mu} \theta-\frac{1}{4} \theta \theta \bar{\theta} \bar{\theta} \square \bar{\phi}(x)
$$

so that

$$
\int \mathrm{d}^{2} \theta \mathrm{d}^{2} \bar{\theta} \bar{\Phi} \Phi=\partial_{\mu} \phi^{*} \partial^{\mu} \phi+\mathrm{i} \bar{\psi} \sigma^{\mu} \partial_{\mu} \psi+F F^{*}
$$

and essentially $\mathcal{G}, \mathcal{K}_{1,2}$ map (2.15) for $\Phi^{(1,2)}$ into

$$
\begin{aligned}
& \left(1-\sum_{n} \frac{1}{n !}\left(\partial^{\mu} \phi^{(1)^{*}} \partial_{\mu} \phi^{(1)}\right)^{n}\right)+\left(1-\sum_{n} \frac{1}{n !}\left(\mathrm{i} \bar{\psi}^{(1)} \sigma^{\mu} \partial_{\mu} \psi^{(1)}\right)^{n}\right) \\
+ & \square^{-1}\left(1-\sum_{n} \frac{1}{n !}\left(F^{(1)^{*}} \square F^{(1)}\right)^{n}\right) \\
+ & \sum_{n, k} \frac{1}{k !}(n \square)^{k}\left(\partial^{\mu} \phi^{(2)^{*}} \partial_{\mu} \phi^{(2)}\right)+\sum_{n, k} \frac{1}{k !}(n \square)^{k}\left(\mathrm{i} \bar{\psi}^{(2)} \sigma^{\mu} \partial_{\mu} \psi^{(2)}\right) \\
+ & \sum_{n, k} \frac{1}{k !}(n \square)^{k}\left(F^{(2)^{*}} F^{(2)}\right) .
\end{aligned}
$$

Thus, as an example, a suitable set of functions in (2.12) is as follows:

$$
\mathcal{K}^{1}(t)=\mathcal{K}^{2}(t)=t
$$

with $t$ generic variable of these functions, i.e., they correspond to minimal Kähler potentials $\Phi^{(1,2)^{\dagger}} \Phi^{(1,2)}$. Moreover, one has

$$
\begin{aligned}
\mathcal{G}\left(\mathcal{X}^{(1)}, \mathcal{Y}^{(1)}, \mathcal{Z}^{(1)}, \mathcal{X}^{(2)}, \mathcal{Y}^{(2)}, \mathcal{Z}^{(2)}\right) & =\left(\mathrm{e}^{\mathcal{X}^{(1)}}-1\right)+\left(\mathrm{e}^{\mathcal{Y}^{(1)}}-1\right)+\square^{-1}\left(\mathrm{e}^{\mathcal{Z}^{(1)}}-1\right) \\
& +\mathcal{F}_{1}\left(\mathcal{X}^{(2)}\right)+\mathcal{F}_{2}\left(\mathcal{Y}^{(2)}\right)+\mathcal{F}_{3}\left(\mathcal{Z}^{(2)}\right)
\end{aligned}
$$

where $\mathcal{F}_{1,2,3}$ are obtained from the following power series:

$$
\mathcal{F}_{1,2,3}\left(\mathcal{X}^{(2)}, \mathcal{Y}^{(2)}, \mathcal{Z}^{(2)}\right)=\sum_{k, n=0}^{\infty} \frac{n^{k}}{k !} \square^{k}\left(\mathcal{X}^{(2)}, \mathcal{Y}^{(2)}, \square^{-1} \mathcal{Z}^{(2)}\right)
$$

5 Naturally, the prescription for $\mathcal{K}^{2}$ encodes the extra sign in the nonlocal kinetic terms for the auxiliary fields, $\mathcal{A}$ in (2.1), with respect to the standard fields' term $\mathcal{F}$. 
while

$$
\begin{aligned}
& \mathcal{X}^{(1,2)} \equiv \partial^{\mu} \phi^{(1,2)^{*}} \partial_{\mu} \phi^{(1,2)}, \\
& \mathcal{Y}^{(1,2)} \equiv \mathrm{i} \bar{\psi}^{(1,2)} \sigma^{\mu} \partial_{\mu} \psi^{(1,2)}, \\
& \mathcal{Z}^{(1,2)} \equiv F^{(1,2)^{*}} \square F^{(1,2)}
\end{aligned}
$$

\section{A. Feynman rules}

In such a model, simple Feynman rules can be consistently defined, as a trivial extension of what holds for ordinary Feynman diagrams. For more details about important technical aspects in the quantization procedure see Appendix E, and for readers who do not feel satisfied as yet, we recall that Refs. [26 30] have considered these features in nonlocal (nonsupersymmetric) gauge theories.

The vertices are unchanged, and the smeared propagator for the fields $\phi^{1}, \psi^{1}$ reads as

$$
\frac{\mathrm{i} \mathcal{E}^{2}}{\left(b^{1}+\mathrm{i} \epsilon\right)}, \frac{\mathrm{i} \mathcal{E}^{2}}{\left(f^{1}+\mathrm{i} \epsilon\right)} \text {. }
$$

Moreover, the smeared propagators for the auxiliary ${ }^{6}$ fields $\phi^{2}, \psi^{2}$ are

$$
\frac{\mathrm{i}\left[I-\mathcal{E}^{2}\right]}{\left(b^{1}+\mathrm{i} \epsilon\right)}, \frac{\mathrm{i}\left[I-\mathcal{E}^{2}\right]}{\left(f^{1}+\mathrm{i} \epsilon\right)} .
$$

On considering the familiar form of the differential operators

$$
b_{1}=\partial^{2}-m^{2}, \quad f_{1}=\mathrm{i} \gamma^{\mu} \partial_{\mu}+m,
$$

the corresponding rules in momentum space are as follows.

i) For the $\phi^{1}, \psi^{1}$ propagator:

$$
\mathrm{i} \frac{\exp \left(\frac{-p^{2}-m^{2}}{\Lambda^{2}}\right)}{\left(p^{2}+m^{2}+\mathrm{i} \epsilon\right)}, \quad \mathrm{i} \frac{\exp \left(\frac{-p^{2}-m^{2}}{\Lambda^{2}}\right)}{\left(\gamma_{\mu} p^{\mu}-m+\mathrm{i} \epsilon\right)},
$$

ii) For the $\phi^{2}, \psi^{2}$ propagator:

$$
\mathrm{i} \frac{\left[I-\exp \left(\frac{-p^{2}-m^{2}}{\Lambda^{2}}\right)\right]}{\left(p^{2}+m^{2}+\mathrm{i} \epsilon\right)}, \mathrm{i} \frac{\left[I-\exp \left(\frac{-p^{2}-m^{2}}{\Lambda^{2}}\right)\right]}{\left(\gamma_{\mu} p^{\mu}-m+\mathrm{i} \epsilon\right)} .
$$

\footnotetext{
${ }^{6}$ Also in this case, as usual in quantum field theory, the Feynman rules for Majorana fermions are reduced to Dirac fermion ones, because of the cancellations between the charge conjugation $C$ operators occurring in the Majorana propagator and in the vertices involving it. For this reason, we omit the presence of the $C$ operators in the given rules.
} 
Equations (2.26) and (2.27) provide the integrand in the integral representation of the propagator ${ }^{7}$. We note, incidentally, that such an integrand is the inverse of the symbol

$$
\frac{\left(p^{2}+m^{2}\right)}{\left(I-\mathrm{e}^{\frac{\left(-p^{2}-m^{2}\right)}{\Lambda^{2}}}\right)} .
$$

We are therefore dealing with pseudodifferential operators, a topic well known in the mathematical literature [44 46].

\section{CONSISTENCY OF THE MODEL}

In this section we discuss the consistency of our model. In particular, we construct a nonlocal quantum field theory which is unitary to all orders of perturbation theory and is invariant under local symmetry transformations. Indeed, in general a nonlocal theory respects causality and unitarity only at tree level. However, we will show how supersymmetry automatically guarantees causality and unitarity to all orders of perturbation theory ${ }^{8}$.

\section{A. Symmetry transformations}

Generically, a nonlocal quantum field theory seems hopeless: how to construct a local gauge symmetry? However, gauge symmetry can be encoded in a nonlocal theory with a new nonlinear transformation rule. In fact, as shown in Ref. [29] for the scalar theory, if an infinitesimal transformation $\delta \phi_{i}^{1}=T_{i}\left[\phi^{1}\right]$ generates a symmetry of the local action $S\left[\phi^{1}\right]$, then a transformation $\hat{\delta} \phi_{i}^{1}=\mathcal{E}_{i j}^{2} T_{j}\left[\phi^{1}+\phi^{2}\left[\phi^{1}\right]\right]$ generates a symmetry for the corresponding nonlocal action $\hat{S}[\phi]$. In a broad sense, the procedure for obtaining a nonlocal theory preserves a deformed version of the usual continuous symmetry, and we can write

$$
\begin{gathered}
\hat{\delta} \phi_{i}^{2}\left[\phi^{1}\right]=\left(I-\mathcal{E}^{2}\right)_{i j} T_{j}\left[\phi^{1}+\phi^{2}\left[\phi^{1}\right]\right]-K_{i j}\left[\phi^{1}+\phi^{2}\left[\phi^{1}\right]\right] \frac{\delta T_{k}}{\delta \phi_{j}^{1}}\left[\phi^{1}+\phi^{2}\left[\phi^{1}\right]\right] \mathcal{E}_{k l}^{2} \frac{\delta \hat{S}\left[\phi^{1}\right]}{\delta \phi_{l}^{1}}, \\
K_{i j}^{-1}\left[\phi^{1}\right]=b_{i j}^{2}-\frac{\delta^{2} \mathcal{I}[\phi]}{\delta \phi_{i} \delta \phi_{j}}
\end{gathered}
$$

\footnotetext{
${ }^{7}$ We would like to note that one can also redefine all propagators with an exchange sign in the exponent of $\mathcal{E}$ : as we will see, this corresponds to introduce polinomial divergences through ordinary fields $\phi^{(1)}, \psi^{(1)}$ rather than through $\phi^{(2)}, \psi^{(2)}$.

${ }^{8}$ Details about the consistency of functional-integral quantization in a nonlocal field theory are discussed in Ref. [29], especially the problem of the measure in such functional integrals.
} 
For our supersymmetric model, this property remains unaffected, in a well understood way, exactly as it occurs in the usual supersymmetric gauge models.

\section{B. Unitarity and causality}

Unitarity is necessary for the consistency and the calculability of a model. This means that the total probability of processes studied in quantum field theory has to be conserved and equal to one. In other words, the $S$-matrix has to satisfy the condition

$$
\mathcal{S S}^{\dagger}=\mathcal{S}^{\dagger} \mathcal{S}=I
$$

On the other hand, causality is fundamental for an obvious reason: without it, a quantum field theory model could never make any predictions testable against observation. Imagine a theory without causality: in order to calculate the probability of a collision occurring, for example, at LHC, we would have to know the status of all the rest of the Universe! In other words, causality is strictly connected to the cluster decomposition principle of the S-matrix [47]: if multi-particle processes $a_{1} \rightarrow b_{1}, a_{2} \rightarrow b_{2}, \ldots, a_{N} \rightarrow b_{N}$ are studied in $N$ laboratories

at spacetime positions $x_{1, . ., N}$ causally disconnected, for which therefore $\left(x_{i}-x_{j}\right)^{2}<0(i \neq j$, $i, j=1, \ldots, N)$, then the S-matrix is factorized into $N$ parts according to

$$
\mathcal{S}_{b_{1}+b_{2}+\ldots b_{N}, a_{1}+a_{2}+\ldots+a_{N}}=\mathcal{S}_{b_{1} a_{1}} \mathcal{S}_{b_{2} a_{2}} \ldots \mathcal{S}_{b_{N} a_{N}} .
$$

From (3.4), we are able to say that quantum fields are commuting at spacelike distances: the causality violation condition is expressed as

$$
[\phi(x), \phi(y)]=0, \quad(x-y)^{2}<0,
$$

which is re-expressed as a microcausality condition [48] on the S-matrix as

$$
\frac{\delta}{\delta \phi(x)}\left(\frac{\delta \mathcal{S}[\phi]}{\delta \phi(y)} \mathcal{S}^{\dagger}[\phi]\right)=0, \quad x \lesssim y
$$

The S-matrix can be written as a Dyson expansion [48] with respect to the couplings $k(x)$ considered as spacetime fields, i.e.,

$$
\mathcal{S}[k(x)]=I+\sum_{n=1}^{\infty} \frac{1}{n !} \int \mathrm{d} x_{1} \ldots \mathrm{d} x_{n} T\left\{\mathcal{S}_{n}\left(x_{1}, \ldots, x_{n}\right) k\left(x_{1}\right) \ldots k\left(x_{n}\right)\right\} .
$$


The conditions (3.3) and (3.6) can be also expressed in terms of a functional Legendre transform mapping $\phi(x)$ into $k(x)$, leading to exactly the same expressions but in the variables $k(x)$.

By setting $k(x)$ equal to a constant $k$, we revert to the usual couplings and we can insert the expansion (3.7) into (3.3). We obtain the following recursive relations for perturbation theory [31]:

$$
\mathcal{C}_{n}=\mathrm{i} \mathcal{S}_{n+1}\left(y, x_{1}, \ldots, x_{n}\right)+\mathrm{i} \sum_{0 \leq k \leq n-1} \mathcal{P}\left\{\mathcal{S}_{k+1}\left(y, x_{1}, . ., x_{k}\right) \mathcal{S}_{n-k}^{\dagger}\left(x_{k+1}, \ldots, x_{n}\right)\right\}
$$

and

$$
\mathcal{S}_{n}\left(x_{1}, \ldots, x_{n}\right)+\mathcal{S}_{n}^{\dagger}\left(x_{1}, \ldots, x_{n}\right)+\sum_{1 \leq k \leq n-1} \mathcal{P}\left\{\mathcal{S}_{k}\left(x_{1}, . ., x_{k}\right) \mathcal{S}_{n-k}^{\dagger}\left(x_{k+1}, \ldots, x_{n}\right)\right\}=0
$$

where

$$
\mathcal{S}_{n}=\int \mathcal{S}_{n}\left(x_{1}, \ldots, x_{n}\right) \mathrm{d} x_{1} \ldots \mathrm{d} x_{n}
$$

and $\mathcal{P}\{\}$ is the sum over all partitions of $\left\{x_{1}, \ldots, x_{n}\right\}$ into $k$ and $n-k$ elements. For example, the simplest is $\left\{x_{1}, . ., x_{k}\right\},\left\{x_{k+1}, . ., x_{n}\right\}$.

Thus, for the first two orders, the causality condition is described by [31]

$$
\begin{gathered}
\mathcal{C}_{1}(x, y)=\mathrm{i}\left[\mathcal{S}_{2}(x, y)+\mathcal{S}_{1}(x) \mathcal{S}_{1}^{\dagger}(y)\right]=0 \\
\mathcal{C}_{2}(x, y)=\mathrm{i}\left[\mathcal{S}_{3}(x, y, z)+\mathcal{S}_{1}(x) \mathcal{S}_{2}^{\dagger}(y, z)+\mathcal{S}_{2}(x, y) \mathcal{S}_{1}^{\dagger}(z)+\mathcal{S}_{2}(x, z) \mathcal{S}_{1}^{\dagger}(y)\right]=0
\end{gathered}
$$

On the other hand, unitarity is expressed by [31]

$$
\begin{gathered}
\mathcal{S}_{1}(x)+\mathcal{S}_{1}^{\dagger}(x)=0 \\
\mathcal{S}_{2}(x, y)+\mathcal{S}_{2}^{\dagger}(x, y)+\mathcal{S}_{1}(x) \mathcal{S}_{1}^{\dagger}(y)+\mathcal{S}_{1}(y) \mathcal{S}_{1}^{\dagger}(x)=0
\end{gathered}
$$

Relations (3.11)-(3.12) and (3.13)-(3.14) can be used as a test of causality and unitarity to all orders of perturbation theory. For instance, by considering the integral relations corresponding to (3.11)-(3.12) one can write

$$
\begin{gathered}
\mathcal{C}_{1}=\int \mathrm{d}^{4} x \mathrm{~d}^{4} y\left[\theta\left(x_{0}-y_{0}\right) \mathcal{C}_{1}(x, y)+\theta\left(y_{0}-x_{0}\right) \mathcal{C}_{1}(y, x)\right]=0, \\
\mathcal{C}_{2}=\int \mathrm{d}^{4} x \mathrm{~d}^{4} y \mathrm{~d}^{4} z \mathcal{C}_{2}(x, y, z) \theta\left(x_{0}-y_{0}\right) \theta\left(y_{0}-z_{0}\right)+5 \text { symmetric terms }=0 .
\end{gathered}
$$


As a consequence, we will use the following criterion in the next section as a signal of causality violation: a momentum dependence in $\mathcal{S}_{1}$, implying a momentum dependence in $\mathcal{C}_{1}$, is surely a signal of causality violation and unitarity violation. In fact, if the amplitude is proportional to a function of the Mandelstam variables $c^{n} / \Lambda^{2}$ with $n>1(c=s, t, u)$ for $c>\Lambda^{2}$ a breakdown of unitarity and causality will occur, leading to an inconsistent theory up to the scale $\Lambda$. In order to arrive to this conclusion, it is not important to perform Lehmann-Szymanszik-Zimmermann transformations in these recursive conditions: the violations will be manifest by the momentum dependence.

\section{SUPERGRAPHS AND CANCELLATIONS OF DIVERGENCES}

In this section, we study perturbation theory in supergraphs formalism rather than ordinary Feynman diagrams. For an useful reference in the ordinary supersymmetric case, see Refs. [33, 49 51] We would like to give prescriptions in order to calculate superfield Green's functions in a nonlocal model:

$$
<0\left|\mathcal{T}\left\{\Phi^{(1,2)}\left(z^{1}\right) \ldots . \Phi^{(1,2)}\left(z^{r}\right) \Phi^{(1,2)^{\dagger}}\left(z^{r+1}\right) \ldots \Phi^{(1,2)^{\dagger}}\left(z^{s}\right)\right\}\right| 0>,
$$

where $z^{r}=\left(x^{r}, \theta^{r}, \bar{\theta}^{r}\right)$ are superspace coordinates, $\Phi^{(1)}$ and $\Phi^{(2)}$ are ordinary and auxiliary superfields, respectively. The propagator is the basic building block of a generic perturbation theory, and we can construct it by propagators of its component fields:

$$
\begin{aligned}
<0\left|\mathcal{T}\left\{\Phi^{(1,2)}(y, \theta) \Phi^{(1,2)}\left(y^{\prime} \theta^{\prime}\right)\right\}\right| 0> & =<0 \mid \mathcal{T}\left\{\left[\phi^{(1,2)}(y)+\sqrt{2} \psi^{(1,2)}(y) \theta+F^{(1,2)} \theta \theta\right]\right. \\
& \left.\times\left[\phi^{(1,2)}\left(y^{\prime}\right)+\sqrt{2} \psi^{(1,2)}\left(y^{\prime}\right) \theta^{\prime}+F^{(1,2)} \theta^{\prime} \theta^{\prime}\right]\right\} \mid 0>,
\end{aligned}
$$

that we can expand, and the only nonvanishing terms are

$$
\begin{aligned}
& \theta \theta<0\left|\mathcal{T}\left\{F^{(1,2)}(y) \phi^{(1,2)}\left(y^{\prime}\right)\right\}\right| 0>+\theta^{\prime} \theta^{\prime}<0\left|\mathcal{T}\left\{\phi^{(1,2)}(y) F^{(1,2)}\left(y^{\prime}\right)\right\}\right| 0> \\
+ & 2 \theta^{\prime \beta} \theta^{\alpha}<0\left|\mathcal{T}\left\{\psi_{\alpha}^{(1,2)}(y) \psi_{\beta}^{(1,2)}\left(y^{\prime}\right)\right\}\right| 0>,
\end{aligned}
$$

with $y, y^{\dagger}$ defined, as usual, as $\left(y, y^{\dagger}\right)=x \pm \mathrm{i} \theta \sigma \bar{\theta}$. The $\phi^{(1,2)}, F^{(1,2)}, \psi^{(1,2)}$ propagators contain $\Delta_{F}^{(1,2)}\left(x-x^{\prime}\right)$ in the form

$$
\begin{aligned}
& <0\left|\mathcal{T}\left\{\psi_{\alpha}^{(1,2)}(x) \psi^{(1,2)^{\beta}}\left(x^{\prime}\right)\right\}\right| 0>\equiv \mathrm{i} \delta_{\alpha}^{\beta} m \Delta_{F}^{(1,2)}\left(x-x^{\prime}\right), \\
& <0\left|\mathcal{T}\left\{\bar{\psi}^{(1,2)^{\dot{\alpha}}}(x) \bar{\psi}_{\dot{\beta}}^{(1,2)}\left(x^{\prime}\right)\right\}\right| 0>\equiv \mathrm{i} \delta_{\dot{\beta}}^{\dot{\alpha}} m \Delta_{F}^{(1,2)}\left(x-x^{\prime}\right),
\end{aligned}
$$




$$
\begin{aligned}
&< 0\left|\mathcal{T}\left\{\psi_{\alpha}^{(1,2)}(x) \bar{\psi}_{\dot{\beta}}^{(1,2)}\left(x^{\prime}\right)\right\}\right| 0>\equiv \sigma_{\alpha \dot{\beta}}^{\mu} \partial_{\mu} \Delta_{F}^{(1,2)}\left(x-x^{\prime}\right), \\
&<0\left|\mathcal{T}\left\{\phi^{(1,2)}(x) \phi^{(1,2)^{*}}\left(x^{\prime}\right)\right\}\right| 0>\equiv \mathrm{i} \Delta_{F}^{(1,2)}\left(x-x^{\prime}\right), \\
&<0\left|\mathcal{T}\left\{\phi^{(1,2)}(x) F^{(1,2)}\left(x^{\prime}\right)\right\}\right| 0>\equiv-\mathrm{i} m \Delta_{F}^{(1,2)}\left(x-x^{\prime}\right), \\
&<0\left|\mathcal{T}\left\{\phi^{(1,2)^{*}}(x) F^{(1,2)^{*}}\left(x^{\prime}\right)\right\}\right| 0>\equiv-\mathrm{i} m \Delta_{F}^{(1,2)}\left(x-x^{\prime}\right), \\
&<0\left|\mathcal{T}\left\{F^{(1,2)}(x) F^{(1,2)^{*}}\left(x^{\prime}\right)\right\}\right| 0>\equiv \mathrm{i} \square \Delta_{F}^{(1,2)}\left(x-x^{\prime}\right) .
\end{aligned}
$$

By substituting these definitions of propagators into (4.3) , we obtain $-\mathrm{i} m \Delta_{F}^{(1,2)}\left(y-y^{\prime}\right)(\theta-$ $\left.\theta^{\prime}\right)^{2}$, whereas

$$
\begin{aligned}
<0 \mid \mathcal{T}\left\{\Phi^{(1,2)}(x, \theta, \bar{\theta})\right. & \left.\Phi^{(1,2)}\left(x^{\prime}, \theta^{\prime}, \bar{\theta}^{\prime}\right)\right\} \mid 0>\equiv-\mathrm{i} m \delta\left(\theta-\theta^{\prime}\right) \exp \left[\mathrm{i}\left(\theta \sigma^{\mu} \bar{\theta}-\theta^{\prime} \sigma^{\mu} \bar{\theta}^{\prime}\right) \partial_{\mu}\right] \Delta_{\mathrm{F}}^{(1,2)}\left(\mathrm{x}-\mathrm{x}^{\prime}\right) \\
& <0\left|\mathcal{T}\left\{\Phi^{(1,2)^{\dagger}}(x, \theta, \bar{\theta}) \Phi^{(1,2)^{\dagger}}\left(x^{\prime}, \theta^{\prime}, \bar{\theta}^{\prime}\right)\right\}\right| 0> \\
\equiv & -\mathrm{i} m \delta\left(\theta-\theta^{\prime}\right) \exp \left[-\mathrm{i}\left(\theta \sigma^{\mu} \bar{\theta}-\theta^{\prime} \sigma^{\mu} \bar{\theta}^{\prime}\right) \partial_{\mu}\right] \Delta_{\mathrm{F}}^{(1,2)}\left(\mathrm{x}-\mathrm{x}^{\prime}\right)
\end{aligned}
$$

and similarly we can construct

$$
<0\left|\mathcal{T}\left\{\Phi^{(1,2)}(x . \theta, \bar{\theta}) \Phi^{(1,2)^{\dagger}}\left(x^{\prime} \cdot \theta^{\prime}, \bar{\theta}^{\prime}\right)\right\}\right| 0>\equiv \operatorname{iexp}\left[\mathrm{i}\left(\theta \sigma^{\mu} \bar{\theta}+\theta^{\prime} \sigma^{\mu} \bar{\theta}^{\prime}-2 \theta \sigma^{\mu} \bar{\theta}^{\prime}\right) \partial_{\mu}\right] \Delta_{F}^{(1,2)}\left(x-x^{\prime}\right) .
$$

The two-point functions $\Delta_{F}^{(1,2)}$ read as

$$
\begin{gathered}
\Delta_{F}^{(1)}=\frac{1}{\left(\square-m^{2}\right)} \mathrm{e}^{\frac{\left(\square^{2}-m^{2}\right)}{\Lambda^{2}}}, \\
\Delta_{F}^{(2)}=\frac{1}{\left(\square-m^{2}\right)}\left(I-\mathrm{e}^{\frac{\left(\square^{2}-m^{2}\right)}{\Lambda^{2}}}\right),
\end{gathered}
$$

according to definitions given in Section II A. Note that 44.14,4.15) are encoding not only an usual free-field propagator, but an infinite number of derivatives resulting from higher order powers of the Kahler terms $\int \mathrm{d}^{2} \theta \mathrm{d}^{2} \bar{\theta} \mathcal{K}^{(1,2)^{a b}} \Phi_{a}^{(1,2)^{\dagger}} \Phi_{b}^{(1,2)}$, i.e., $\phi^{(1,2) *} \square^{n} \phi^{(1,2)}$ and $\bar{\psi}^{(1,2)}\left(\sigma_{\mu} \partial^{\mu}\right)^{n} \psi^{(1,2)}$ in terms of scalar and fermion components of the chiral field. In a nonlocal model, it is more natural and simpler to consider these higher-derivative interactions just in effective smeared propagators, as (4.14.4.15).

With these prescriptions for propagators, we can evaluate all superfields' Green functions, to any order of perturbation theory. The $n$-th order contribution is

$$
<0\left|\mathcal{T}\left\{\Phi\left(z^{1}\right) \ldots \Phi^{\dagger}\left(z^{r+1}\right) \ldots \int \mathcal{L}_{\text {int }}\left(x_{1}^{\prime}\right) \mathrm{d}^{4} x_{1}^{\prime} \ldots \int \mathcal{L}_{\text {int }}\left(x_{n}^{\prime}\right) \mathrm{d}^{4} x_{n}^{\prime}\right\}\right| 0>
$$


with

$$
\mathcal{L}_{\text {int }}=\int \mathrm{d}^{2} \theta_{n} \mathrm{~d}^{2} \bar{\theta}_{n}^{2} \frac{1}{3}\left[g \Phi^{3}\left(x_{n}^{\prime}, \theta_{n}, \bar{\theta}_{n}\right) \delta\left(\bar{\theta}_{n}\right)+g^{*}\left(\Phi^{\dagger}\left(x_{n}^{\prime}, \theta_{n}, \bar{\theta}_{n}\right)\right)^{3} \delta\left(\bar{\theta}_{n}\right)\right]
$$

that we can evaluate through Feynman diagrams, using Wick's theorem. In the following subsections, we will show explicit applications of this perturbative machinery to a nonlocal supersymmetric model.

Superfields' Green functions are related to the generating functional $Z[J]$ by

$$
\begin{aligned}
& \mathcal{G}^{n}\left(z^{1}, \ldots, z^{m} ; z^{m+1}, \ldots, z^{n}\right) \\
& =(-\mathrm{i})^{n}\left[\frac{\delta}{\delta J^{(1,2)}\left(z^{1}\right)} \cdots \frac{\delta}{\delta J^{(1,2)}\left(z^{m}\right)} \frac{\delta}{\delta J^{(1,2)^{\dagger}}\left(z^{m+1}\right)} \cdots \frac{\delta}{\delta J^{(1,2)^{\dagger}\left(z^{n}\right)}} Z\left[J^{(1,2)}, J^{(1,2)^{\dagger}}\right]\right]_{J^{(1,2)}=J^{(1,2)^{\dagger}}=0}(4.18)
\end{aligned}
$$

where

$$
\begin{gathered}
Z\left[J^{(1,2)}, J^{\left.(1,2)^{\dagger}\right]}=\exp \left\{\mathrm{i} \int d^{4} x \mathcal{L}_{\mathrm{int}}\left(\frac{\delta}{\delta J^{(1,2)}}, \frac{\delta}{\delta J^{(1,2)^{\dagger}}}\right)\right\} Z_{0}\left[J^{(1,2)}, J^{(1,2)^{\dagger}}\right]\right. \\
Z_{0}\left[J^{(1,2)}, J^{\left.(1,2)^{\dagger}\right]}=\exp \left[-\frac{\mathrm{i}}{2} \int \mathrm{d}^{4} x \mathrm{~d}^{2} \theta \mathrm{d}^{4} x^{\prime} \mathrm{d}^{4} \theta^{\prime}\left(J^{(1,2)}(z), J^{(1,2)^{\dagger}}(z)\right) \Delta_{N L}^{(1,2}\left(J^{(1,2)}(z), J^{(1,2)^{\dagger}}(z)\right)^{T}\right]\right. \\
\Delta_{N L}^{(1)}=\frac{1}{\left(\square-m^{2}\right)} \mathrm{e}^{\frac{\square-m^{2}}{\Lambda^{2}}}\left(\begin{array}{cc}
\frac{D^{2}}{\square} & I \\
I & \frac{\bar{D}^{2}}{\square}
\end{array}\right) \delta\left(z-z^{\prime}\right) \\
\Delta_{N L}^{(2)}=\frac{1}{\left(\square-m^{2}\right)}\left[I-\mathrm{e}^{\frac{\square-m^{2}}{\Lambda^{2}}}\right]\left(\begin{array}{cc}
\frac{D^{2}}{\square} & I \\
I & \frac{\bar{D}^{2}}{\square}
\end{array}\right) \delta\left(z-z^{\prime}\right)
\end{gathered}
$$

Now we are ready to display Feynman diagrams for supergraphs in nonlocal Wess-Zumino, according to the following recipe:

(i) Write a chiral field $\Phi_{1}$ (in-going) or $\bar{\Phi}$ (out-going) for any external line.

(ii) For each vertex $\Phi^{(1,2)} \Phi^{(1,2)} \Phi^{(1,2)}$ (and $\left.\bar{\Phi}^{(1,2)} \bar{\Phi}^{(1,2)} \bar{\Phi}^{(1,2)}\right)$ write a $-\frac{1}{4} \bar{D}^{2}\left(\frac{1}{4} D^{2}\right.$ ) acting on one internal propagator.

(iii) Write $\frac{1}{3} g$ (couplings) for each vertex $\left(\frac{1}{3} g^{*}\right.$ for a vertex of antichiral fields' interactions).

(iv) Use propagators (4.21) for each internal line of ordinary chiral (antichiral fields) and (4.22) for each internal line of auxiliary fields.

(v) Compute the combinatory factor and integrate for each vertex as $\int \mathrm{d}^{2} \theta \mathrm{d}^{4} x$ (in the antichiral case write instead $\left.\mathrm{d}^{2} \bar{\theta}\right)$. 
a)

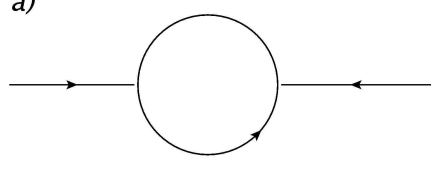

d)

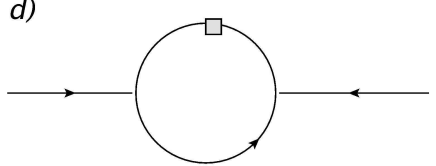

g)

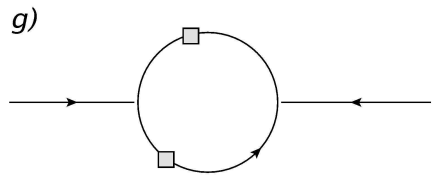

b)

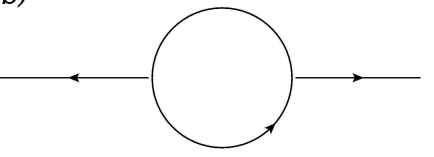

e)

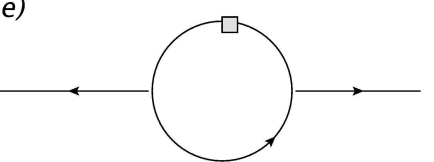

h)

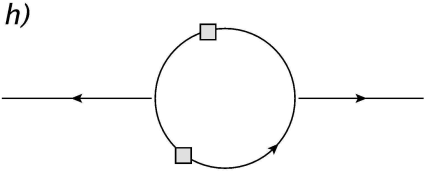

c)

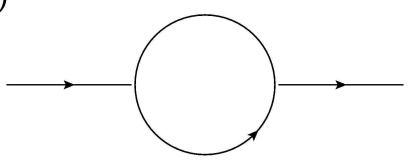

f)

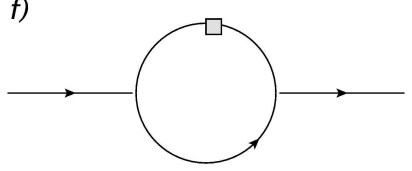

i)

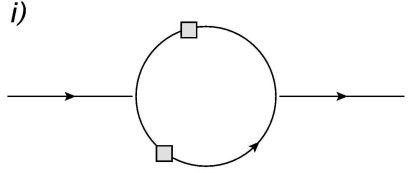

Figure 1: Radiative corrections to the two-point functions (superfield propagators) of $\Phi^{(1)} \Phi^{(1)}$, $\Phi^{(1)^{\dagger}} \Phi^{(1)^{\dagger}}$ and $\Phi^{(1)} \Phi^{(1)^{\dagger}}$. Diagrams (a)-(b)-(c) are radiative corrections to $\Phi^{(1)} \Phi^{(1)}$ two-point functions, with contributions resulting not only from $\Phi^{(1)} \Phi^{(1)}$ internal lines, but also from auxiliary fields $\Phi^{(2)} \Phi^{(2)}$. Diagrams (d)-(e)-(f) are corrections to $\Phi^{(1)^{\dagger}} \Phi^{(1)^{\dagger}}$ with internal lines $\Phi^{(1,2)^{\dagger}} \Phi^{(1,2)^{\dagger}}$. Diagrams (g)-(h)-(i) are corrections to $\Phi^{(1)} \Phi^{(1)^{\dagger}}$ by internal lines $\Phi^{(1,2)} \Phi^{(1,2)^{\dagger}}$. The only diagrams different from zero are (g)-(h)-(i), the other ones are all automatically vanishing.

In the following sections, we will apply to superfield propagators our technique for evaluation of radiative corrections, couplings and tadpoles. Eventually, we will consider scattering processes.

\section{A. Radiative corrections to superfield propagators}

Let us consider one-loop radiative corrections to superfield two-point functions. The possible diagrams in a nonlocal Wess-Zumino model are shown in Fig. 1. All integrals (a)-(b)-(c)-(d)-(e)-(f) are automatically vanishing: vertices in (a)-(b)-(c) are proportional to $\delta^{2}\left(\theta-\theta^{\prime}\right)=\delta(0)$ which is set to 0 in dimensional regularization; vertices in (d)-(e)-(f) are proportional to $\mathrm{d}^{2} \bar{\theta}$ and $\mathrm{d}^{2} \bar{\theta}^{\prime}$, i.e., they are proportional to $\delta^{2}\left(\bar{\theta}-\bar{\theta}^{\prime}\right)=\delta(0)$ which is set to 0 with the same understanding. As a consequence, we obtain the following important result, that can be simply generalized to $n$ loops: all contributions to mass renormalization are vanishing also in nonlocal supersymmetric models. Let us now consider the contributions of 
(g)-(h)-(i) in Fig. 1:

$$
\begin{aligned}
& \mathcal{I}_{g}=\int \mathrm{d}^{4} x \mathrm{~d}^{4} x^{\prime} \mathrm{d}^{2} \theta \mathrm{d}^{2} \theta^{\prime} \mathrm{d}^{2} \bar{\theta} \mathrm{d}^{2} \bar{\theta}^{\prime} \delta(\bar{\theta}) \delta\left(\theta^{\prime}\right) \Phi^{(1)}(x, \theta, \bar{\theta}) \exp \left[\mathrm{i}\left(\theta \sigma^{\mu} \bar{\theta}+\theta^{\prime} \sigma^{\mu} \bar{\theta}^{\prime}-2 \theta \sigma^{\mu} \bar{\theta}^{\prime}\right) \partial_{\mu}\right] \\
& \times \Delta_{F}^{(1)}\left(x-x^{\prime}\right) \exp \left[\mathrm{i}\left(\theta \sigma^{\mu} \bar{\theta}+\theta^{\prime} \sigma^{\mu} \bar{\theta}^{\prime}-2 \theta \sigma^{\mu} \bar{\theta}^{\prime}\right) \partial_{\mu}\right] \Delta_{F}^{(1)}\left(x-x^{\prime}\right) \Phi^{(1)^{\dagger}}\left(x^{\prime}, \theta^{\prime}, \bar{\theta}^{\prime}\right), \\
& \mathcal{I}_{h}=\int \mathrm{d}^{4} x \mathrm{~d}^{4} x^{\prime} \mathrm{d}^{2} \theta \mathrm{d}^{2} \theta^{\prime} \mathrm{d}^{2} \bar{\theta} \mathrm{d}^{2} \bar{\theta}^{\prime} \delta(\bar{\theta}) \delta\left(\theta^{\prime}\right) \Phi^{(1)}(x, \theta, \bar{\theta}) \exp \left[\mathrm{i}\left(\theta \sigma^{\mu} \bar{\theta}+\theta^{\prime} \sigma^{\mu} \bar{\theta}^{\prime}-2 \theta \sigma^{\mu} \bar{\theta}^{\prime}\right) \partial_{\mu}\right], \\
& \times \Delta_{F}^{(1)}\left(x-x^{\prime}\right) \exp \left[\mathrm{i}\left(\theta \sigma^{\mu} \bar{\theta}+\theta^{\prime} \sigma^{\mu} \bar{\theta}^{\prime}-2 \theta \sigma^{\mu} \bar{\theta}^{\prime}\right) \partial_{\mu}\right] \Delta_{F}^{(2)}\left(x-x^{\prime}\right) \Phi^{(1)^{\dagger}}\left(x^{\prime}, \theta^{\prime}, \bar{\theta}^{\prime}\right), \\
& \mathcal{I}_{i}=\int \mathrm{d}^{4} x \mathrm{~d}^{4} x^{\prime} \mathrm{d}^{2} \theta \mathrm{d}^{2} \theta^{\prime} \mathrm{d}^{2} \bar{\theta} \mathrm{d}^{2} \bar{\theta}^{\prime} \delta(\bar{\theta}) \delta\left(\theta^{\prime}\right) \Phi^{(1)}(x, \theta, \bar{\theta}) \exp \left[\mathrm{i}\left(\theta \sigma^{\mu} \bar{\theta}+\theta^{\prime} \sigma^{\mu} \bar{\theta}^{\prime}-2 \theta \sigma^{\mu} \bar{\theta}^{\prime}\right) \partial_{\mu}\right] \\
& \times \Delta_{F}^{(2)}\left(x-x^{\prime}\right) \exp \left[\mathrm{i}\left(\theta \sigma^{\mu} \bar{\theta}+\theta^{\prime} \sigma^{\mu} \bar{\theta}^{\prime}-2 \theta \sigma^{\mu} \bar{\theta}^{\prime}\right) \partial_{\mu}\right] \Delta_{F}^{(2)}\left(x-x^{\prime}\right) \Phi^{(1)^{\dagger}}\left(x^{\prime}, \theta^{\prime}, \bar{\theta}^{\prime}\right) .
\end{aligned}
$$

These integrals are contributing to the corrections of the wave functions. In the limit of $\Lambda \rightarrow \infty$, these integrals give logaritmic divergences, that can be absorbed in the wave function renormalization. In this case, we are integrating extra exponential factors of the momenta, suppressing divergences in (4.23), but reintroducing wild divergences (i.e., out of control) in the integral (4.25). In particular, an infinite power series of momenta is expected, leading to a nonrenormalizable theory. However, these extra corrections $p^{2 n}$ are suppressed as $\Lambda^{-2 n}$ at the $n$-th order. Thus, nonrenormalizzable contributions can be handled and controlled if $\Lambda>>\bar{E}$, where $\bar{E}$ is the energy scale observed in laboratories. Of course, nonrenormalizzable contributions are expected to be controlled by a theory beyond a nonlocal quantum field model, bearing in mind string theory as a natural completion of our model. Anyway, the importance of supersymmetry is manifest in the automatic cancellations of infinitely many infinities originating from Figs. 1-(a)-(b)-(c)-(d)-(e)-(f).

\section{B. Tadpoles and corrections to coupling constants}

In this section, we consider possible tadpoles contributions and corrections to coupling constants, in our nonlocal model. Without supersymmetry, it is generically expected that these contributions are wildly divergent as an infinite power series of the momenta. We show

all relevant contributions in Fig. 2. However, in supersymmetry, supergraphs' formalism shows manifestly that all tadpoles cancel each other, because all diagrams are proportional to $\delta(0)$. As a consequence, we obtain again a cancellation of an infinite number of infinities 

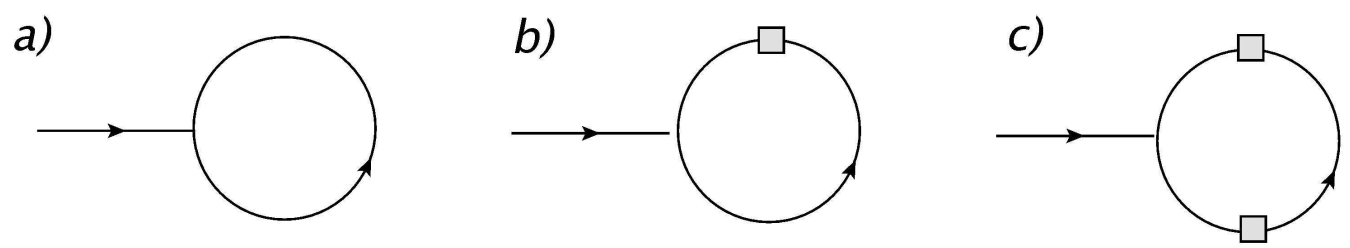

Figure 2: Tadpole diagrams resulting from $\Phi^{(1,2)}$ (ordinary and auxiliary fields).
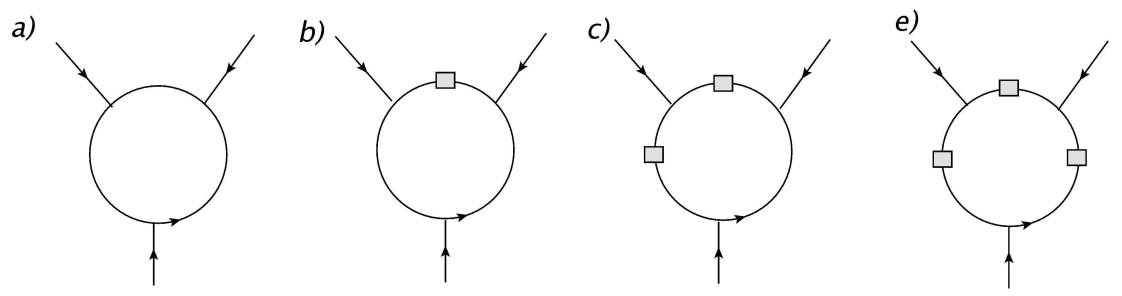

Figure 3: Corrections to $\Phi^{3}$ originating from $\Phi^{(1,2)}$ (ordinary and auxiliary fields). All these diagrams are vanishing. Similarly, also diagrams with all arrows going out $\left(\Phi^{\dagger}\right)^{3}$ are automatically equal to zero.

just by virtue of supersymmetry. In this case, there are no other possible tadpoles not cancelled by supersymmetry.

Similarly, also possible corrections to superpotential terms $\Phi^{3}$ (Fig.3) and $\bar{\Phi}^{3}$ are identically zero because they are again proportional to $\delta(0)$.

\section{ONE-LOOP ACAUSAL DIAGRAMS IN $\phi \phi \rightarrow \phi \phi$ SCATTERING}

In this section, we reconsider the same acausal one-loop process in a $\lambda\left(\phi^{(1)}\right)^{4}$ scalar self-interacting model, evaluated in Ref. [31]. As shown in this reference, the scattering $\phi^{(1)} \phi^{(1)} \rightarrow \phi^{(1)} \phi^{(1)}$ at one loop has a divergent amplitude (see Fig. 4). The harmful contribution results from the auxiliary field $\phi^{(2)}$ propagating inside the loops (off-shell) and interacting with $\phi^{(1)}$ as interaction terms $\sim\left(\phi^{(1)}\right)^{3} \phi^{(2)}$. By assuming the massless case $m=0$, the (renormalized) amplitude is [31, 32]

$$
\mathcal{A}(s, t, u)_{\text {Boson }}=\frac{9 \lambda^{2}}{4 \pi^{2}} \sum_{c=s, t, u} \sum_{n=0}^{\infty} \frac{\left(\frac{c}{\Lambda^{2}}\right)^{n}\left(1-\frac{1}{2^{n}}\right)}{n((n+1) !)},
$$

where $c=s, t, u$ are the Mandelstam variables, and we are summing over the three channels. We express this as a power-series expansion, because of the nonlinear functions on the vertices. 
As usual in quantum field theory, we can choose the Schwinger parametrization and we can rewrite the amplitudes as the following integrals for the $s$-channel bosonic part (see Ref. [31, 32]):

$$
\mathcal{A}(s)_{\mathrm{B}}=\frac{9 \lambda^{2}}{4 \pi^{2}} \int_{0}^{1 / 2} \mathrm{~d} x \int_{\frac{1}{(1-x)}}^{\frac{1}{x}} \frac{\mathrm{d} \zeta}{\zeta} \exp \left\{-\frac{\zeta}{\Lambda^{2}}\left(m^{2}-x(1-x) s\right)\right\} .
$$

The asymptotic expansion of this integral in the neighboorhood of $s=0$ reads as

$$
\mathcal{A}_{B}(s) \sim \sum_{n=0}^{2} a_{n}(m, \Lambda) s^{n}+\mathrm{O}\left(s^{3}\right)
$$

where the coefficients $a_{0,1,2}$ are given by

$$
\begin{gathered}
a_{0}(m, \Lambda)=\frac{9 \lambda^{2}}{4 \pi^{2}} \int_{0}^{1 / 2} \mathrm{~d} x \int_{\frac{1}{(1-x)}}^{\frac{1}{x}} \frac{\mathrm{d} \zeta}{\zeta} \mathrm{e}^{-\frac{m^{2} \zeta}{\Lambda^{2}}} \\
a_{1}(m, \Lambda)=\frac{9 \lambda^{2}}{4 \pi^{2}} \int_{0}^{1 / 2} \mathrm{~d} x \int_{\frac{1}{(1-x)}}^{\frac{1}{x}} \mathrm{~d} \zeta \frac{x(1-x)}{\Lambda^{2}} \mathrm{e}^{-\frac{m^{2} \zeta}{\Lambda^{2}}} \\
a_{2}(m, \Lambda)=\frac{9 \lambda^{2}}{4 \pi^{2}} \int_{0}^{1 / 2} \mathrm{~d} x \int_{\frac{1}{(1-x)}}^{\frac{1}{x}} \zeta \mathrm{d} \zeta \frac{x^{2}(1-x)^{2}}{2 \Lambda^{4}} \mathrm{e}^{-\frac{m^{2} \zeta}{\Lambda^{2}}} .
\end{gathered}
$$

As we said for the massless case, the zeroth and first order of the expansion (5.3) are constants cancelled in the renormalization (subtraction of counterterms). The result can be rewritten as

$$
\mathcal{A}_{B}(s, t, u)=\frac{9 \lambda^{2}}{4 \pi^{2}} \sum_{c=s, t, u}\left[-\log \left[c / \Lambda^{2}\right]+\sum_{n=0}^{\infty} \frac{1-2^{-n}}{(n+1) ! n}\left(\frac{c}{\Lambda^{2}}\right)^{n}\right],
$$

where the first logarithmic contributions are the analogous of the local QFT result. [52]:

We also report evaluation of the diagram in Fig. 4 (the first one therein), calculated in

$$
\mathcal{A}_{B}(s, t, u)=\frac{9 \lambda^{2}}{8 \pi^{2}} \sum_{c=s, t, u}\left[-\log \frac{c}{\Lambda^{2}}-2 \sum_{n=1}^{\infty} \frac{1}{n !(n+1)}\left(1-\frac{1}{2^{n+1}}\right)\left(\frac{c}{\Lambda^{2}}\right)^{n}\right],
$$

where the first logarithmic contributions are the analogous of the local quantum field theory result.

However, in a nonlocal Wess-Zumino model, we have to consider also other diagrams resulting from the fermionic sector. In fact, we have to consider carefully all possible oneloop diagrams involving also all interactions between auxiliary superfields and standard superfields: Yukawa like terms $\phi^{(2)} \bar{\psi}_{L}^{(1)} \psi_{R}^{(1)}+$ h.c., $\phi^{(1)} \bar{\psi}_{L}^{(2)} \psi_{R}^{(1)}+$ h.c.; interactions in the 


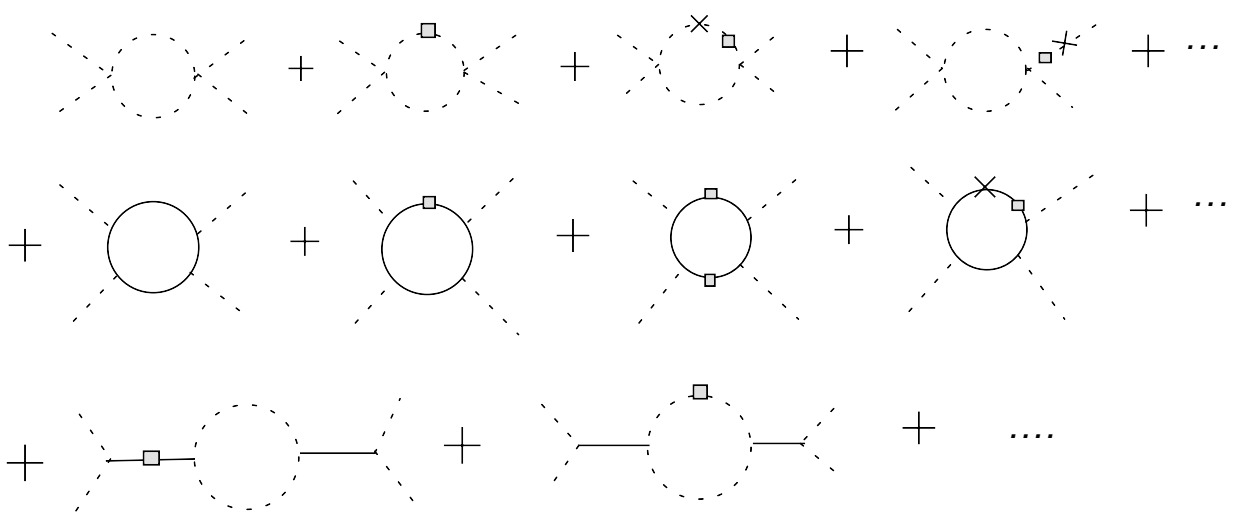

Figure 4: $\phi^{1} \phi^{1} \rightarrow \phi^{1} \phi^{1}$ : One-loop diagrams leading to violations of causality. Dashed lines represent scalars $\phi^{1,2}$, continuous lines describe fermions $\psi^{1,2}$. Boxes on the lines represent auxiliary fields $\phi^{2}$ and $\psi^{2}$ (dashed and continuous, respectively). Bilinear mixing terms between standard fields and auxiliary ones are represented as crosses. Other, trivial complications of these diagrams are not reported. By virtue of supersymmetry, all diagrams, with all entering arrows, cancel each other.

scalars' sector $\left(\phi^{(1)}\right)^{3}\left(\phi^{(2)}\right)$ and $\left(\phi^{(1)}\right)^{2}\left(\phi^{(2)}\right)^{2}$ etc., mass-mixing terms $m \bar{\psi}_{L}^{(1)} \psi^{(2)}+$ h.c. etc. The computation might become quite tedious if we were to consider all numerical prefactors in the Taylor power series expansions inside the integrals and the complications from trace operations, the presence of particles' masses etc. But we would like to note that all parameters in the diagrams are connected by supersymmetry, and the fermionic contributions are opposite in sign with respect to the bosonic ones. Thus, from a naive power-counting, it seems reasonable that some cancellations will occur in this case. Now let us switch our language from ordinary Feynman diagrams to supergraphs. In Fig.5, relevant one-loop supergraphs are reported. The evaluation of all these diagrams seems unfeasible. However, we can conclude that all diagrams in Fig. 5, except (d), are equal to zero because all these diagrams are proportional to $\delta(0)$, resulting from internal $\Phi^{1,2} \Phi^{1,2}$ and $\bar{\Phi}^{1,2} \bar{\Phi}^{1,2}$. In 
fact, we have the following amplitudes:

$$
\begin{aligned}
& \mathcal{A}_{1}=C_{1} \frac{g^{4}}{12^{4}} \iiint \int \mathrm{d}^{4} x_{1,2,3,4} \mathrm{~d}^{2} \theta_{1,2,3,4} \mathrm{~d} \Phi\left(z_{1}\right) \Phi\left(z_{2}\right)\left(\bar{D}^{2}\right)\left[\frac{1}{\left(\square-m^{2}\right)} \mathrm{e}^{\frac{\square-m^{2}}{\Lambda^{2}}}\right]_{z_{1}, z_{2}} \\
& \times\left(\bar{D}^{2}\right)\left[\frac{1}{\left(\square-m^{2}\right)} \mathrm{e}^{\frac{\square-m^{2}}{\Lambda^{2}}}\right]_{z_{2}, z_{3}}\left(\bar{D}^{2}\right)\left[\frac{1}{\left(\square-m^{2}\right)} \mathrm{e}^{\frac{\square-m^{2}}{\Lambda^{2}}}\right]_{z_{3}, z_{4}} \\
& \times\left(\bar{D}^{2}\right)\left[\frac{1}{\left(\square-m^{2}\right)} \mathrm{e}^{\frac{\square-m^{2}}{\Lambda^{2}}}\right]_{z_{4}, z_{1}} \Phi\left(z_{3}\right) \Phi\left(z_{4}\right) \\
& \times \delta\left(z_{1}-z_{2}\right) \delta\left(z_{2}-z_{3}\right) \delta\left(z_{3}-z_{4}\right) \delta\left(z_{4}-z_{1}\right), \\
& \mathcal{A}_{2}=C_{2} \frac{g^{4}}{12^{4}} \iiint \int \mathrm{d}^{4} x_{1,2,3,4} \mathrm{~d}^{2} \theta_{1,2,3,4} \Phi\left(z_{1}\right) \Phi\left(z_{2}\right)\left(\bar{D}^{2}\right)\left[\frac{1}{\left(\square-m^{2}\right)}\left(I-\mathrm{e}^{\frac{\square-m^{2}}{\Lambda^{2}}}\right)\right]_{z_{1}, z_{2}} \\
& \times\left(\bar{D}^{2}\right)\left[\frac{1}{\left(\square-m^{2}\right)} \mathrm{e}^{\frac{\square-m^{2}}{\Lambda^{2}}}\right]_{z_{2}, z_{3}}\left(\bar{D}^{2}\right)\left[\frac{1}{\left(\square-m^{2}\right)} \mathrm{e}^{\frac{\square-m^{2}}{\Lambda^{2}}}\right]_{z_{3}, z_{4}} \\
& \times\left(\bar{D}^{2}\right)\left[\frac{1}{\left(\square-m^{2}\right)} \mathrm{e}^{\frac{\square-m^{2}}{\Lambda^{2}}}\right]_{z_{4}, z_{1}} \Phi\left(z_{3}\right) \Phi\left(z_{4}\right) \\
& \times \delta\left(z_{1}-z_{2}\right) \delta\left(z_{2}-z_{3}\right) \delta\left(z_{3}-z_{4}\right) \delta\left(z_{4}-z_{1}\right) \\
& \mathcal{A}_{3}=C_{3} \frac{g^{4}}{12^{4}} \iiint \int \mathrm{d}^{4} x_{1,2,3,4} \mathrm{~d}^{2} \theta_{1,2,3,4} \Phi\left(z_{1}\right) \Phi\left(z_{2}\right)\left(\bar{D}^{2}\right)\left[\frac{1}{\left(\square-m^{2}\right)}\left(I-\mathrm{e}^{\frac{\square-m^{2}}{\Lambda^{2}}}\right)\right]_{z_{1}, z_{2}} \\
& \times\left(\bar{D}^{2}\right)\left[\frac{1}{\left(\square-m^{2}\right)}\left(I-\mathrm{e}^{\frac{\square-m^{2}}{\Lambda^{2}}}\right)\right]_{z_{2}, z_{3}}\left(\bar{D}^{2}\right)\left[\frac{1}{\left(\square-m^{2}\right)} \mathrm{e}^{\frac{\square-m^{2}}{\Lambda^{2}}}\right]_{z_{3}, z_{4}} \\
& \times\left(\bar{D}^{2}\right)\left[\frac{1}{\left(\square-m^{2}\right)} \mathrm{e}^{\frac{\square-m^{2}}{\Lambda^{2}}}\right]_{z_{4}, z_{1}} \Phi\left(z_{3}\right) \Phi\left(z_{4}\right) \\
& \times \delta\left(z_{1}-z_{2}\right) \delta\left(z_{2}-z_{3}\right) \delta\left(z_{3}-z_{4}\right) \delta\left(z_{4}-z_{1}\right) \\
& \mathcal{A}_{4}=C_{4} \frac{g^{4}}{12^{4}} \iiint \int \mathrm{d}^{4} x_{1,2,3,4} \mathrm{~d}^{2} \theta_{1,2,3,4} \Phi\left(z_{1}\right) \Phi\left(z_{2}\right)\left(\bar{D}^{2}\right)\left[\frac{1}{\left(\square-m^{2}\right)}\left(I-\mathrm{e}^{\frac{\square-m^{2}}{\Lambda^{2}}}\right)\right]_{z_{1}, z_{2}} \\
& \times\left(\bar{D}^{2}\right)\left[\frac{1}{\left(\square-m^{2}\right)}\left(I-\mathrm{e}^{\frac{\square-m^{2}}{\Lambda^{2}}}\right)\right]_{z_{2}, z_{3}}\left(\bar{D}^{2}\right)\left[\frac{1}{\left(\square-m^{2}\right)}\left(I-\mathrm{e}^{\frac{\square-m^{2}}{\Lambda^{2}}}\right)\right]_{z_{3}, z_{4}} \\
& \times\left(\bar{D}^{2}\right)\left[\frac{1}{\left(\square-m^{2}\right)} \mathrm{e}^{\frac{\square-m^{2}}{\Lambda^{2}}}\right]_{z_{4}, z_{1}} \Phi\left(z_{3}\right) \Phi\left(z_{4}\right) \\
& \times \delta\left(z_{1}-z_{2}\right) \delta\left(z_{2}-z_{3}\right) \delta\left(z_{3}-z_{4}\right) \delta\left(z_{4}-z_{1}\right) \\
& \mathcal{A}_{5}=C_{5} \frac{g^{4}}{12^{4}} \iiint \int \mathrm{d}^{4} x_{1,2,3,4} \mathrm{~d}^{2} \theta_{1,2,3,4} \Phi\left(z_{1}\right) \Phi\left(z_{2}\right)\left(\bar{D}^{2}\right)\left[\frac{1}{\left(\square-m^{2}\right)}\left(I-\mathrm{e}^{\frac{\square-m^{2}}{\Lambda^{2}}}\right)\right]_{z_{1}, z_{2}} \\
& \times\left(\bar{D}^{2}\right)\left[\frac{1}{\left(\square-m^{2}\right)}\left(I-\mathrm{e}^{\frac{\square-m^{2}}{\Lambda^{2}}}\right)\right]_{z_{2}, z_{3}}\left(\bar{D}^{2}\right)\left[\frac{1}{\left(\square-m^{2}\right)}\left(I-\mathrm{e}^{\frac{\square-m^{2}}{\Lambda^{2}}}\right)\right]_{z_{3}, z_{4}} \\
& \times\left(\bar{D}^{2}\right)\left[\frac{1}{\square-m^{2}}\left(I-\mathrm{e}^{\frac{\square-m^{2}}{\Lambda^{2}}}\right)\right]_{z_{4}, z_{1}} \Phi\left(z_{3}\right) \Phi\left(z_{4}\right) \\
& \times \delta\left(z_{1}-z_{2}\right) \delta\left(z_{2}-z_{3}\right) \delta\left(z_{3}-z_{4}\right) \delta\left(z_{4}-z_{1}\right) \text {. }
\end{aligned}
$$


Let us bear in mind the following useful relations:

$$
\frac{1}{16} \frac{\bar{D}^{2} D^{2}}{\square} \Phi=\Phi, \quad \bar{D} \frac{1}{16} \frac{\bar{D}^{2} D^{2}}{\square} \Phi=0, \quad \mathrm{~d}^{2} \theta=-\frac{1}{4} \bar{D}^{2},
$$

the last being valid inside the integral $\int \mathrm{d}^{4} x . C_{1,2,3,4,5}$ are combinatorial factors. Amplitudes $\mathcal{A}_{6,7,8,9,10}$ in Fig. 5-(b) have the same form of $\mathcal{A}_{1,2,3,4,5}$ respectively, with suitable substitutions $\Phi^{(1,2)} \rightarrow \Phi^{(1,2)^{\dagger}}$ and $\bar{D} \rightarrow D$. In this form, cancellations are made explicit by the fact that the number of $\bar{D}\left(\mathrm{~d}^{2} \theta\right)$ is not equal to the number of $D\left(\mathrm{~d}^{2} \bar{\theta}\right)$ in these amplitudes, and this leads to $\delta(0)$ s inside the integral, as mentioned above. As far as $\mathcal{A}_{11,15}$ in Fig. 5-(c) is concerned, as well as $\mathcal{A}_{11,15}$ but with reversed arrows (not reported in Fig. 5), the number of $D$ is different by the one of $\bar{D}$, so we have cancellations of these contributions. Note that among these contributions discussed here, there are not only $F$-terms, but also some possible $D$-terms (three entering arrows, one going out; three going out, one entering; two entering, two going out). As a cascade, a $n$-loop generalization of this result is understood. We would like to remark that also in this case an infinite number of divergences are automatically cancelled. This reflects the fact that fermionic and bosonic components, as the ones reported in Fig. 4 (the possible contributions are not all written down in this figure) are canceling each other.

Examples of cancellations just shown are not only accidental properties of one-loop calculations, but they are expected at all orders of perturbation theory. In fact, in a nonlocal supersymmetric model, the nonrenormalization theorem remains valid. This crucial point will be discussed in the next sections. By virtue of these powerful properties we will conclude that all $F$-terms' processes discussed above are not corrected by an infinite number of divergences.

\section{ACAUSALITY CANCELLATIONS IN F-TERMS TO ALL ORDERS OF PER- TURBATION THEORY}

In the particular case studied in Sec. IV, we are considering the quantum one-loop corrections of a supersymmetric F-term. In fact, the interaction $\lambda\left(\phi^{1}\right)^{4}$ is contained in the superpotential and obtained after the integration on the Grassmannian (see Secs. IA and II). For this reason, we can say that the cancellations must occur to all orders of perturbation theory, by virtue of the nonrenormalization theorem [33, 34] of supersymmetry (see Appendix). This tells us that in a supersymmetric theory the cancellation of radiative 

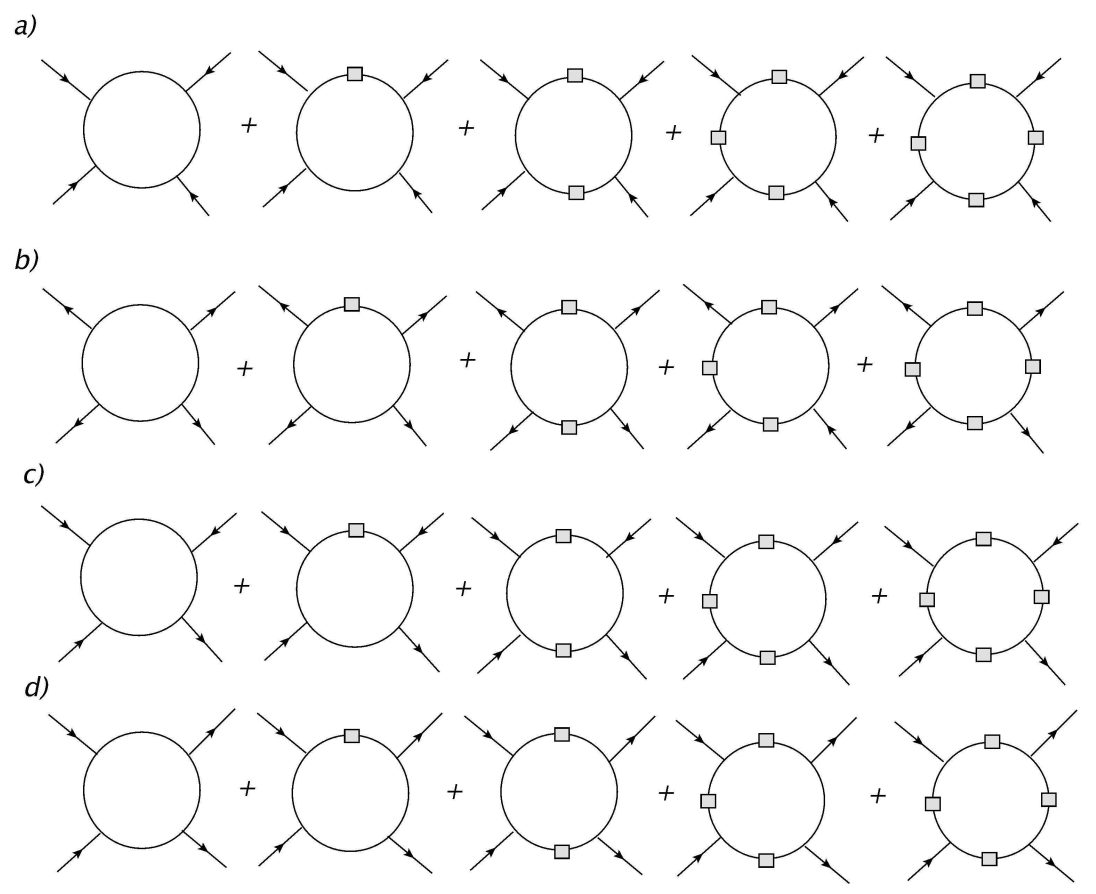

Figure 5: One-loop supergraphs contributions to $\phi^{(1)} \phi^{(1)} \rightarrow \phi^{(1)} \phi^{(1)}$. In these diagrams, propagators are all $\Phi^{(1,2)} \Phi^{(1,2)}$ in (a), all $\bar{\Phi}^{(1,2)} \bar{\Phi}^{(1,2)}$ in (b), one $\Phi^{(1,2)} \Phi^{(1,2)}$ and three $\bar{\Phi}^{(1,2)} \Phi^{(1,2)}$ in (c); one $\Phi^{(1,2)} \Phi^{(1,2)}$, one $\bar{\Phi}^{(1,2)} \bar{\Phi}^{(1,2)}$ and two $\bar{\Phi}^{(1,2)} \Phi^{(1,2)}$ in (d). By virtue of supersymmetry, all these diagrams are equal to zero, except (d).

corrections occurs to all orders, and is a consequence of the holomorphic nature of the superpotential $\mathcal{W}\left(\Phi^{1}+\Phi^{2}\right)$, not permitting a perturbative renormalization of the supersymmetric $F$-terms. Thus, we have the following peculiar result: a nonlocal $\lambda \phi^{4}$ model is not consistent without supersymmetry, but with the help of supersymmetry we automatically obtain a consistent and perfectly computable example of a nonlocal supersymmetric quantum field theory without acausalities or other inconsistencies. Note that, in our nonlocal formulation, we have never modified the holomorphic superpotential, but only the Kähler potential (Dterms). For this reason, the nonrenormalization proof given by Seiberg [34] is still valid. In the next subsection, we summarize Seiberg's argument about the Wess-Zumino case with comments about its relations with locality ${ }^{9}$.

\footnotetext{
${ }^{9}$ We would like to note that the nonlocal Wess-Zumino class of models under discussion is invariant under the superPoincaré group. As mentioned in the introduction, nonlocal theories violating the Poincaré invariance are not included in our considerations. For example, a Wess-Zumino model in noncommutative geometry is an example of a nonlocal supersymmetric quantum field theory, violating the local
} 


\section{A. Seiberg's argument about Wess-Zumino theory, relaxing locality}

Consider an initial (tree-level) superpotential (B7). In the case $m=\lambda=0$, we are restoring a global symmetry $G=U(1) \times U(1) \times U(1)_{R}$. Such a global symmetry can be defined also in a nonlocal field theory in the same way of a local one. The field $\Phi$ transforms as $(1,1)$ under $G$. This constrains the dimensions of the couplings, i.e., $\operatorname{dim}(m) \in(-2,0)$ and $\operatorname{dim}(\lambda) \in(-3,-1)$. A general superpotential invariant under $G$ can be written as

$$
\mathcal{W}_{\mathrm{eff}}=m \Phi^{2} f\left(\frac{\lambda \Phi}{m}\right),
$$

where $f$ is a generic holomorphic function. We can always consider a power-series expansion of $f$. The $n$-th term invariant under the global group $G$ is $c_{n} \Phi^{n}$, where $c_{n}(\lambda, m)=\lambda^{n-2} \frac{1}{m^{n-3}}$. But this is also the same result obtained from a tree graph with exchanges of the superfields $\Phi$. These have not to occur in the Wilsonian effective action; higher orders in $\lambda$ to the coefficient $c_{n}(\lambda, m)$ cannot be considered in the general structure of (6.1). Thus, we conclude that the resulting effective superpotential is exactly equal to (6.1), and it is not renormalized.

In general, we can ask ourselves whether, by relaxing the hypothesis of locality, this simple argument can be avoided. However, we want to stress that in the nonlocal formulation of the Wess-Zumino model given in Sec. II, we have never modified the superpotentials, we have just deformed the Kähler potentials. The argument is based only upon the holomorphic nature of the superpotentials, and in the nonlocal formulation this property is not affected. Thus, we conclude that Seiberg's argument remains valid also in a nonlocal Wess-Zumino $\operatorname{model}^{10}$.

superPoincaré group. In this case, the nonrenormalization theorem does not protect the F-terms from quantum corrections. On the other hand, as shown in Ref. 53], there remains also in this case a residual supersymmetry $(\mathcal{N}=1 / 2)$ protecting the F-terms from an infinite number of quantum corrections.

10 A generalization to other cases given in Ref. 34] can be considered. For example, we can study a supersymmetric quantum chromodynamics with a superpotential $S \bar{Q} Q+\lambda^{\prime} S^{3}$, with $S$ a gauge singlet of $U(1)_{S}$ (local gauge symmetry generalized as in section IIIA). Upon taking $\lambda=\lambda^{\prime}=0$, the theory is invariant under $G=S U\left(N_{f}\right)_{L} \times S U\left(N_{f}\right)_{R} \times U(1)_{V} \times U(1)_{S} \times U(1)_{R}$. We can then repeat an argument similar to the Wess-Zumino case, as shown in Ref. 34]. 

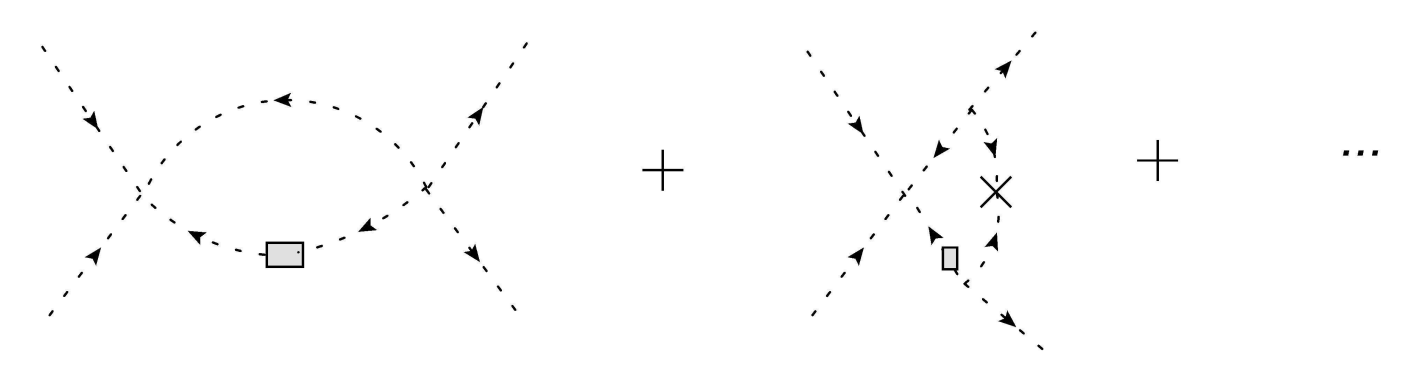

Figure 6: $\phi^{(1)} \phi^{(1)} \rightarrow \phi^{(1)} \phi^{(1)}$ : we report two examples of one-loop diagrams not protected by the nonrenormalization theorem in nonlocal Wess-Zumino model. These are ordinary Feynman diagrams for the scalar sector.

\section{B. Acausal corrections of $D$-terms in nonlocal Wess-Zumino}

In Wess-Zumino models, $F$-term superpotential terms are protected by acausal radiative corrections. We report contributions to $\phi \phi \rightarrow \phi \phi$. Diagrams in Fig. 5-(d) lead to acausalities. In Fig. 6, we give two examples of contributions from the scalar sector, with ordinary Feynman diagrams. These diagrams have divergences, violating causality and unitarity for $s, t, u>\Lambda$. The amplitude of the first one in Fig. 6, for example, is exactly equal to (5.2). As a consequence, not all possible acausal diagrams are cancelled, but infinitely many are cancelled just by virtue of supersymmetry, as discussed in the previous section. Consider the integral

$$
\begin{aligned}
\tilde{\mathcal{A}}_{2} & =\tilde{C}_{2} \frac{g^{4}}{12^{4}} \iiint \int \mathrm{d}^{4} x_{1} \mathrm{~d}^{2} \theta_{1} \mathrm{~d}^{4} x_{2} \mathrm{~d}^{2} \bar{\theta}_{2} \mathrm{~d}^{4} x_{3} \mathrm{~d}^{2} \bar{\theta}_{3} \mathrm{~d}^{4} x_{4} \mathrm{~d}^{2} \theta_{4} \Phi\left(z_{1}\right) \bar{\Phi}\left(z_{2}\right)\left[\frac{1}{\left(\square-m^{2}\right)} \mathrm{e}^{\frac{\square-m^{2}}{\Lambda^{2}}}\right]_{z_{1}, z_{2}} \\
& \times D^{4} \bar{D}^{4}\left[\frac{1}{\left(\square-m^{2}\right)} \mathrm{e}^{\frac{\square-m^{2}}{\Lambda^{2}}}\right]_{z_{2}, z_{3}}\left[\frac{1}{\left(\square-m^{2}\right)} \mathrm{e}^{\frac{\square-m^{2}}{\Lambda^{2}}}\right]_{z_{3}, z_{4}}\left[\frac{1}{\left(\square-m^{2}\right)}\left(I-\mathrm{e}^{\frac{\square-m^{2}}{\Lambda^{2}}}\right)\right]_{z_{4}, z_{1}} \\
& \times \bar{\Phi}\left(z_{3}\right) \Phi\left(z_{4}\right) \delta\left(z_{1}-z_{2}\right) \delta\left(z_{2}-z_{3}\right) \delta\left(z_{3}-z_{4}\right) \delta\left(z_{4}-z_{1}\right) .
\end{aligned}
$$

In order to evaluate this integral, let us exploit the relations

$$
\int \mathrm{d}^{4} \theta_{n} \bar{D}^{2} D^{2} \delta\left(\theta_{n}-\theta_{1}\right) \delta\left(\bar{\theta}_{n}-\bar{\theta}_{1}\right)=16 \int \mathrm{d}^{4} \theta_{n}
$$

Since we are not particularly interested in all numerical prefactors, in massless approximation, we note that in momentum space this amplitude is approximated by

$$
\tilde{\mathcal{A}}_{2}(p) \sim \int \mathrm{d} p p^{-5} \mathrm{e}^{-3 p^{2} / \Lambda^{2}}\left(I-\mathrm{e}^{p^{2} / \Lambda^{2}}\right) .
$$

From this we can argue that, even if there are divergent contributions, they will be suppressed as $\Lambda^{-n}$ with $n>5$. This result provides further evidence of the power of supersymmetry: 
also acausal divergences become softer than in nonsupersymmetric case by one order of magnitude. Thus, the amplitude calculated gets some cancellations by contributions of the fermionic sector. Not all divergences are cancelled, but up to fifth order in momenta, there are cancellations of $p^{n}$ with $n<5$ (logarithmic divergences remain). Other contributions, like ones with mass insertions, are more suppressed than (6.4).

\section{NONLOCAL SUPER YANG-MILLS MODEL}

We now want to consider a more realistic quantum field theory, generalizing our observations for the Wess-Zumino case to a general supersymmetric Yang-Mills theory. For this purpose, we have to introduce a standard vector supermultiplet $\left\{D^{1}, A_{\mu}^{1}, \lambda^{1}\right\}$ and an auxiliary vector supermultiplet $\left\{D^{2}, A_{\mu}^{2}, \lambda^{2}\right\}$. The nonlocal action is

$$
\begin{aligned}
\hat{S}\left[D^{1}, A^{1}, \lambda^{1}\right] & =\frac{1}{2} \int \mathrm{d}^{4} x\left\{\hat{D}_{c}^{1}\left(a^{1}\right)_{c d} \hat{D}_{d}^{1}+\hat{A}_{c \mu}^{1}\left(b^{1}\right)_{c d}^{\mu \nu} \hat{A}_{d \nu}^{1}-D_{c}^{2}\left(a^{2}\right)_{c d} D_{d}^{2}-\hat{A}_{c \mu}^{2}\left(b^{2}\right)_{c d}^{\mu \nu} \hat{A}_{d \nu}^{2}\right. \\
& \left.+\hat{\lambda}_{a \alpha}^{1}\left(f^{1}\right)_{a b}^{\alpha \dot{\beta}} \hat{\lambda}_{b \dot{\beta}}^{1}-\lambda_{a \alpha}^{2}\left(f^{2}\right)_{a b}^{\alpha \dot{\beta}} \lambda_{b \dot{\beta}}^{2}+\mathcal{I}\left\{A^{1}+A^{2}, \lambda^{1}+\lambda^{2}\right\}+\text { h.c. }\right\}
\end{aligned}
$$

where $D^{2}, A^{2}, \psi^{2}$ are functionals in the variables $D^{1}, A^{1}, \psi^{1}$ and solve the equations

$$
\frac{\delta S}{\delta D^{2}}=\frac{\delta S}{\delta A^{2}}=\frac{\delta S}{\delta \lambda^{2}}=0
$$

$a^{1}, b^{1}, f^{1}$ are the nonlocal operators containing masses and kinetic terms, $a^{2}, b^{2}, f^{2}$ are the nonlocal operators of the auxiliary fields; $a^{2}, b^{2}, f^{2}$ have exactly the same definitions given in Eq. (2.9) and the redefined fields $\hat{D}^{1}, \hat{A}^{1}, \hat{\lambda}^{1}$ can be expressed as in Eqs. (2.4)-(2.8).

The nonlocal gauge symmetry of the action (7.1) is as follows:

$$
\hat{\delta}_{\theta}\left(A^{1}\right)_{a}^{\mu}=\mathcal{E}_{B}^{2}\left\{-\theta_{a}^{, \mu}+g f_{b c d}\left(A_{c \mu}^{1}+A_{c \mu}^{2}\left[A^{1}, \lambda^{1}\right]\right) \theta_{d}\right\}
$$

supplemented by the analogous transformation for the gaugino field $\lambda^{1}$. The nonlocality in infinitesimal gauge transformations like (7.3) results from the functional $A_{2}\left[D^{1}, A_{1}, \lambda_{1}\right]$. We can reformulate the action (7.1) in a form explicitly invariant under supersymmetry. For simplicity, we do not consider Fayet-Iliopoulos terms, that can be inserted in a well understood way. Thus, the supersymmetric action in superspace is

$$
\mathcal{S}_{S Y M}=\int \mathrm{d}^{4} x \mathcal{G}_{S Y M}\left\{\frac{1}{32 \pi} \operatorname{Im}\left[\tau \int \mathrm{d}^{2} \theta \mathrm{d}^{2} \bar{\theta}\left(\mathcal{K}_{\mathrm{NLYM}}\left(W_{\alpha}^{1}, W_{\alpha}^{2}\right)+\text { h.c. }\right)\right]\right\},
$$


where $\mathcal{G}_{N L S Y M}\{\mathcal{O}\}$ and $\mathcal{K}_{\text {NLYM }}$ are realizing the nonlocal deformation of the standard gaugeinvariant term $W^{\alpha} W_{\alpha}$. We can also consider a coupling to a matter chiral superfield as

$$
\begin{array}{r}
\mathcal{S}=\mathcal{S}_{\mathrm{SYM}}+\int \mathrm{d}^{4} x\left(\mathcal{G}_{N L G T}\left\{\int \mathrm{d}^{2} \theta \mathrm{d}^{2} \bar{\theta} \mathcal{K}_{\mathrm{NLM}}\left(\bar{\Phi}_{1}+\bar{\Phi}_{2}, \mathrm{e}^{V_{1}}+\mathrm{e}^{V_{2}}, \Phi_{1}+\Phi_{2}\right)\right\}\right) \\
+\int \mathrm{d}^{4} x\left[\int \mathrm{d}^{2} \theta \mathcal{W}\left(\Phi_{1}+\Phi_{2}\right)+\int \mathrm{d}^{2} \bar{\theta} \overline{\mathcal{W}}\left(\bar{\Phi}_{1}+\bar{\Phi}_{2}\right)\right],
\end{array}
$$

where $\mathcal{K}_{\mathrm{NLM}}$ and $\mathcal{G}_{N L M}\{\mathcal{O}\}$ are extending the standard term $\bar{\Phi}^{V} \Phi$ to a nonlocal one. A function $\mathcal{G}_{N L M}$ similar to the one considered above for Wess-Zumino can be suggested in this case.

\section{A. Acausalities in super Yang-Mills}

In nonlocal supersymmetric Yang-Mills, in BRST quantization, the propagators are exactly the same as the ones just considered in the Wess-Zumino model, but with an extra color factor $\delta_{a b}$ (see, for nonsupersymmetric Yang-Mills, Ref. [29]). Note that we will have also ghost and auxiliary ghost propagators

$$
\begin{gathered}
-\frac{\mathrm{i} \delta_{a b}}{\left(p^{2}-\mathrm{i} \epsilon\right)} \exp \left(-\frac{p^{2}}{\Lambda^{2}}\right)=-\mathrm{i} \delta_{a b} \int_{1}^{\infty} \frac{\mathrm{d} \zeta}{\Lambda^{2}} \exp \left(-\zeta \frac{p^{2}}{\Lambda^{2}}\right), \\
-\frac{\mathrm{i} \delta_{a b}}{\left(p^{2}-\mathrm{i} \epsilon\right)}\left\{1-\exp \left(-\frac{p^{2}}{\Lambda^{2}}\right)\right\}=-\mathrm{i} \delta_{a b} \int_{0}^{1} \frac{d \zeta}{\Lambda^{2}} \exp \left(-\zeta \frac{p^{2}}{\Lambda^{2}}\right),
\end{gathered}
$$

and supersymmetric partners of ghosts, i.e.,

$$
\begin{gathered}
-\frac{\mathrm{i} \delta_{\alpha \dot{\beta}}}{\left(\gamma_{\mu} p^{\mu}-\mathrm{i} \epsilon\right)} \exp \left(-\frac{p^{2}}{\Lambda^{2}}\right), \\
-\frac{\mathrm{i} \delta_{\alpha \beta}}{\left(\gamma_{\mu} p^{\mu}-\mathrm{i} \epsilon\right)}\left\{1-\exp \left(-\frac{p^{2}}{\Lambda^{2}}\right)\right\} .
\end{gathered}
$$

Let us consider, in analogy with the Wess-Zumino case, a scattering $A_{\mu}^{(1)} A_{\mu}^{(1)} \rightarrow A_{\mu}^{(1)} A_{\mu}^{(1)}$. We have to note that now this interaction does not result from the $F$-terms as in WessZumino $\phi^{(1)} \phi^{(1)} \rightarrow \phi^{(1)} \phi^{(1)}$, but from the $D$-terms. This holds despite the fact that the Feynman diagrams involved are exactly similar to Fig. 4 but with the replacements $\phi^{(1,2)} \rightarrow$ $A_{\mu}^{(1,2)}$ and $\psi^{(1,2)} \rightarrow \lambda^{(1,2)}$ (and with extra color gauge factors in the vertices understood), and with other extra contributions resulting from standard ghost supermultiplets and auxiliary ones. 
The nonrenormalization theorem does not protect the $D$-terms from quantum loop corrections. As a consequence, supersymmetry is not the way to cancel acausalities in a generic super Yang-Mills theory. But generally, n-loops' diagrams resulting from the fermionic sector and the bosonic one contribute to the divergences with opposite signs. Thus, we can mitigate the causality problem with a suitable gauge group and fields' content in our theory. For example, with a tuning of the coupling parameters, in principle it could be possible to tune to zero the first relevant divergences in the total amplitudes. However, it is unclear how to achieve a cancellation to all orders in perturbation theory and to all orders of divergences with a finite content of fields. It therefore seems that the only way to obtain a complete cancellation of all acausal divergences in a nonlocal quantum field theory is to introduce an infinite number of fermions and bosons, but also in this framework it remains unclear whether exact cancellations can be proved. On the other hand, we argue that also in this case supersymmetry is protecting the model from acausal contributions in the $F$-terms. For this reason, supersymmetry seems a natural step towards the realization of a completely consistent nonlocal quantum field theory.

\section{CONCLUSIONS AND REMARKS}

The issue of nonlocality in field theory has many important aspects. In ordinary quantum field theory on a flat spacetime background, the assumption of causality restricts the singularities of the $n$-point Green functions to lie within the future tube. This means that the Green functions propagate positive frequencies purely forward in time, and negative frequencies purely backwards. When spacetime is instead curved, this picture may be substantially affected. For example, the Euclidean section of a black-hole metric has nontrivial topology with Euler number 2. This implies that there exists an obstruction to the Wick rotation of the time axis, and hence, when the Euclidean Green functions are analytically continued to the Lorentzian regime, they contain acausal singularities periodically distributed in the

imaginary time coordinate [54]. On the other hand, in a space-of-histories formulation of gauge theories, the connection forms naturally available are nonlocal, since they are defined with the help of Green functions of an invertible operator acting on gauge fields, as is shown in detail in Ref. [55].

In this paper, we have shown how supersymmetry can play a fundamental role for a 
consistent realization of nonlocal field theories. In nonsupersymmetric $\lambda \phi^{4}$ models, acausal contributions occur at one loop in simple processes like $\phi \phi \rightarrow \phi \phi$ scatterings. Also in the Wess-Zumino case, we have acausal divergent diagrams, but the contributions resulting from the bosonic sector and the fermionic sector cancel each other in F-terms, to all orders of perturbation theory. We remark that, in a nonlocal model, this corresponds to a complete cancellation of an infinite number of divergences. We have also noticed that the nonrenormalization theorem of supersymmetry will guarantee us not only an alternative argument to the quantitative calculation given, but also a proof of cancellations to all orders. Unfortunately, the nonrenormalization theorem does not cancel all divergences: quantum corrections of $D$-terms are not protected and acasual divergences are not cancelled in corresponding diagrams. The results have been discussed also for a nonlocal supersymmetric Yang-Mills model, including the case of coupling to matter. Also in this last case, acausal contributions are cancelled in $F$-terms, but in $D$-terms they are generically remaining. The problem therefore arises to prove that, in order to cancel the infinitely many acausal diagrams, one has to introduce an infinite number of bosons and fermions. This is naturally occurring in string theory, generically predicting a Kaluza-Klein tower of modes, also higher spins' ones. It is quite natural to extend the formalism suggested in this paper to the case of higher spins' superfields, in which generically the equations of motion have nonlocal kinetic terms, as for a spin-3 field. Thus, we think that our nonlocal supersymmetry model could be reinterpreted as an effective quantum field theory model of a string theory.

On the other hand, supersymmetry does not occur at energies $E<\mathrm{TeV}$ and in principle, if present, it could be broken near the Planck scale. Thus, nonlocal gauge theories continue to possess acausalities in quantum loops at energies lower than the supersymmetry-breaking scale. Of course, harmful loop diagrams resulting from $F$-terms are cancelled at $\Lambda_{\text {SUSY }}$ when supersymmetry is restored; while the ones originating from $D$-terms are presumably alleviated at a scale lower than $\Lambda$ because of counter cancellations between bosons and fermions; and then completely cancelled at $\Lambda$, a process in which we think that an infinite number of bosons and fermions are excited. As argued in Refs. [31, 32, 52], it is practically impossible to detect such violations if the $\Lambda$-scale of cutoff, introduced in the smeared propagators, is (for example) close to the Planck scale.

Another comment is regarding the problem of quantum gravity. In fact, as we said in the Introduction, practically all main candidates for a quantum theory of gravity are 
predicting the loss of locality at the quantum scale. This is a clear point in order to avoid an undesirable problem like the curvature singularity. As a consequence, it is fundamental to consider a meaningful nonlocal quantum theory of matter, which makes it possible to develop perturbation theory. The present paper might represent a first step towards this goal: a cancellation of an infinite number of acausal infinities for a nonlocal quantum field theory. As far as the underlying classical theory is concerned, it also remains to be seen how to formulate the Cauchy problem, a proper understanding of which is very important in theories of gravity or motivated by gravity [56].

\section{Acknowledgments}

A. A. is grateful to the Galileo Galilei Institute for Theoretical physics for the hospitality, where this paper was prepared. A. A. is particularly grateful to Massimo Bianchi for discussions and suggestions on these subjects. A.A. would also like to thank Zurab Berezhiani, Francisco Morales, Luca Griguolo, Gabriele Veneziano and Anupam Mazumdar for valuable remarks and suggestions. G. E. is grateful to the Dipartimento di Fisica of Federico II University, Naples, for hospitality and support, and to K. Kirsten for correspondence. The authors are indebted to P. Townsend for a careful reading of the manuscript.

\section{Appendix A: The nonrenormalization theorem of supersymmetry}

Since it plays a key role in our analysis, we now present a brief review of the conceptual framework for the nonrenormalization theorem of supersymmetry. A key idea [34] is to think of all coupling constants $\lambda_{i}$ in the superpotential as background chiral fields. The effective superpotential of the dynamical fields $\phi_{I}$ and the background fields $\lambda_{i}$ is subject to the following constraints [34]:

(i) The nonvanishing values of coupling constants are interpreted as spontaneously breaking the global symmetry group $G$. The effective Lagrangian which depends both on $\phi_{I}$ and $\lambda_{i}$ should be invariant under $G$.

(ii) The effective superpotential $W_{\text {eff }}$ is a (locally) holomorphic function of all fields. Since the coupling constants are treated as fields as well, this implies that $W_{\text {eff }}$ is independent of 
the Hermitian conjugates $\lambda_{i}^{\dagger}$, unlike what happens in ordinary field theories.

(iii) The effective superpotential can depend on the dynamically generated scale of the theory, denoted by $\Lambda$, and it should be smooth in the limit $\Lambda \rightarrow 0$. In almost all cases, this implies that $W_{\text {eff }}$ cannot grow faster than $\phi^{3}$, as a field $\phi$ takes increasingly large values. The gauge couplings are asymptotically free.

(iv) The behavior of $W_{\text {eff }}$ when the coupling constants in the bare superpotential approach 0 can be analyzed with perturbative methods, and this constrains the small $\lambda_{i}$ limit. It might happen that there are more light fields at $\lambda_{i}=0$ than at nonvanishing values of $\lambda_{i}$. Upon integrating out these fields and not including them in the effective action, $W_{\text {eff }}$ might be nonanalytic at $\lambda_{i}=0$. This is the peculiar weak-coupling regime.

Interestingly, it turns out that the effective superpotential is not a generic function of the fields consistent with the symmetries. There exist some terms which are consistent with all symmetries of the problem but are not generated by perturbative or nonperturbative effects. This clearly violates the so-called principle of naturalness. The author of Ref. [34] develops heuristic arguments, and assumes that the theory can be regularized while preserving all symmetries.

We would like to note that nonrenormalization theorems of (rigid) supersymmetry are not lost upon relaxing the assumption of locality in a supersymmetric quantum field theory. In fact, SuperPoincaré is a rigid global group of transformations, as a generalization of the Poincaré one. A de-localization of an interaction spacetime point preserves global supersymmetry. As a simple analogy, we consider scattering processes in classical mechanics: in a collision between two, or N, pointlike masses, angular momentum is conserved, as well as in a collision between two, or $\mathrm{N}$, rigid bodies; i.e., the angular momentum theorem is not dependent on pointlike or volume-like interactions. This is a general theorem of classical mechanics, as a consequence of Noether's theorem for translation and rotation global groups, in the Euclidean three-dimensional space. The Lagrangian of classical mechanics is always invariant under the angular momentum operator $\boldsymbol{L}$, as well as the supersymmetry Lagrangian which is also invariant under the $\boldsymbol{Q}$ generator. From this last trivial propriety it suddenly follows, in $\mathcal{N}=1$ supersymmetry, that only $D$ - and $F$-terms can be written. Locality in the interactions is not relevant in this argument, in classical mechanics as well as in supersymmetric quantum field theory. On the other hand, for supergravity, local 
supersymmetry is requested, but a nonlocal supergravity is beyond the aims of this paper.

\section{Appendix B: A brief review of a local Wess-Zumino model}

The Wess-Zumino model is a simple supersymmetric interacting theory of a chiral supermultiplet $\Phi$ (see, for example, Ref. [43]). In the superspace formalism, we can write the Lagrangian of this model in an explicitly supersymmetric form ${ }^{11}$

$$
\begin{gathered}
\mathcal{L}_{\mathrm{WZ}}=\mathcal{L}_{\text {kin }}+\mathcal{L}_{\text {int }}, \\
\mathcal{L}_{\text {kin }}=\int \mathrm{d}^{2} \theta \mathrm{d}^{2} \bar{\theta} \bar{\Phi} \Phi+\text { h.c. } \\
\mathcal{L}_{\text {int }}=\int \mathrm{d}^{2} \theta \mathcal{W}(\Phi)+\int \mathrm{d}^{2} \bar{\theta} \overline{\mathcal{W}}(\bar{\Phi})=-\frac{\delta \mathcal{W}}{\delta \phi} F-\frac{1}{2} \frac{\delta^{2} \mathcal{W}}{\delta \phi^{2}} \psi \psi+\text { h.c. }
\end{gathered}
$$

where

$$
\mathcal{W}(\Phi)=\mathcal{W}(\phi)+\sqrt{2} \frac{\delta \mathcal{W}}{\delta \phi} \theta \psi-\theta \theta\left(\frac{\delta \mathcal{W}}{\delta \phi} F+\frac{1}{2} \frac{\delta^{2} \mathcal{W}}{\delta \phi^{2}} \psi \psi\right)
$$

having denoted by $F$ and $\bar{F}$ the auxiliary fields. After integrating out the auxiliary fields, we obtain

$$
F=\frac{\delta \overline{\mathcal{W}}}{\delta \phi}, \quad \bar{F}=\frac{\delta \mathcal{W}}{\delta \phi}
$$

Upon integrating over the Grassmannian superspace, the total Lagrangian is

$$
\mathcal{L}_{\mathrm{WZ}}=\mathcal{L}_{\text {kin }}+\mathcal{L}_{\text {int }}=\partial_{\mu} \bar{\phi} \partial^{\mu} \phi+\frac{\mathrm{i}}{2} \psi \partial_{\mu} \sigma^{\mu} \psi+\bar{F} F-\frac{\delta \mathcal{W}}{\delta \phi} F-\frac{1}{2} \frac{\delta^{2} W}{\delta \phi^{2}} \psi \psi+\text { h.c. }
$$

For a renormalizable theory the superpotential is

$$
\mathcal{W}\left(\Phi^{i}\right)=\frac{1}{2} m_{i j} \Phi^{i} \Phi^{j}+\frac{1}{3} g_{i j k} \Phi^{i} \Phi^{j} \Phi^{k}
$$

We do not consider linear terms as usually done, but in principle this action can be extended

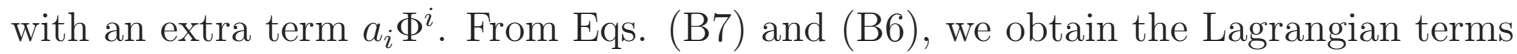

$$
\begin{gathered}
\mathcal{L}_{\text {kin }}=\partial^{\mu} \phi^{*} \partial_{\mu} \phi+\frac{\mathrm{i}}{2} \psi \gamma^{\mu} \partial_{\mu} \psi+\text { h.c. } \\
\mathcal{L}_{\text {int }}=-\left|m \phi+\lambda \phi^{2}\right|^{2}-\frac{1}{2}\left[m\left(\bar{\psi}_{R} \psi_{L}+\bar{\psi}_{L} \psi_{R}\right)+2 \lambda\left(\phi \bar{\psi}_{R} \psi_{L}+\phi^{*} \bar{\psi}_{L} \psi_{R}\right)\right] .
\end{gathered}
$$

\footnotetext{
11 The kinetic term might be generalized to a more generic functional $\mathcal{K}(\Phi, \bar{\Phi})$ called nonminimal Kähler potential.
} 


\section{Appendix C: Local super Yang-Mills model}

We here consider a supersymmetric Yang-Mills model, with locality, of a vector supermultiplet $V$ with a generic gauge group $S U(N)$ (see, for a general reference, Ref. [43]). The Lagrangian is

$\mathcal{L}_{S Y M}=\frac{1}{32 \pi} \operatorname{Im}\left(\tau \int d^{2} \theta W^{\alpha} W_{\alpha}\right)=\operatorname{Tr}\left[-\frac{1}{4} F_{\mu \nu} F^{\mu \nu}-i \lambda \sigma^{\mu} D_{\mu} \bar{\lambda}+\frac{1}{2} D^{2}\right]+\frac{\theta_{Y M}}{32 \pi^{2}} g^{2} \operatorname{Tr} F_{\mu \nu} \tilde{F}^{\mu \nu}$,

where

$$
W_{\alpha}=-\frac{1}{4} \bar{D} \bar{D}\left(\mathrm{e}^{-V} D_{\alpha} \mathrm{e}^{V}\right), \quad \bar{W}_{\dot{\alpha}}=-\frac{1}{4} D D\left(\mathrm{e}^{V} \bar{D}_{\alpha} \mathrm{e}^{-V}\right),
$$

having defined $\tau \equiv \frac{\theta_{Y M}}{2 \pi}+\frac{4 \pi \mathrm{i}}{g^{2}}$ and $\tilde{F}^{\mu \nu} \equiv \frac{1}{2} \epsilon^{\mu \nu \rho \sigma} F_{\rho \sigma}$.

On considering a theory with matter chiral superfields coupled with the vector superfield, the Lagrangian term (B2) is extended as supersymmetric gauge-invariant term $\bar{\Phi} \mathrm{e}^{V} \Phi$,

$$
\mathcal{L}_{\text {matter }}=\int \mathrm{d}^{2} \bar{\theta} \bar{\Phi} \mathrm{e}^{V} \Phi+\int \mathrm{d}^{2} \theta \mathcal{W}(\Phi)+\int \mathrm{d}^{2} \bar{\theta} \bar{W}(\bar{\Phi})
$$

In the Wess-Zumino gauge

$$
\begin{gathered}
\bar{\Phi} \mathrm{e}^{V} \Phi=\bar{\Phi} \Phi+\bar{\Phi} V \Phi+\frac{1}{2} \bar{\Phi} V^{2} \Phi, \\
{[\bar{\Phi} V \Phi]_{\theta \theta \bar{\theta} \bar{\theta}}=\frac{\mathrm{i}}{2} \bar{\phi} A^{\mu} \partial_{\mu} \phi-\frac{\mathrm{i}}{2} \partial_{\mu} \bar{\phi} A^{\mu} \phi-\frac{1}{2} \bar{\psi} \bar{\sigma}^{\mu} A_{\mu} \psi+\frac{\mathrm{i}}{\sqrt{2}} \bar{\phi} \lambda \psi-\frac{\mathrm{i}}{\sqrt{2}} \bar{\psi} \bar{\lambda} \phi+\frac{1}{2} \bar{\phi} D \phi,} \\
{\left[\bar{\Phi} V^{2} \Phi\right]_{\theta \theta \bar{\theta} \bar{\theta}}=\frac{1}{2} \bar{\phi} A^{\mu} A_{\mu} \phi,}
\end{gathered}
$$

and replacing $V \rightarrow 2 g V$, we obtain a Lagrangian

$$
\begin{aligned}
\mathcal{L} & =\mathcal{L}_{\mathrm{SYM}}+\mathcal{L}_{\text {matter }}+\mathcal{L}_{\mathrm{FI}}=\frac{1}{32 \pi} \operatorname{Im}\left(\tau \int \mathrm{d}^{2} \theta W^{\alpha} W_{\alpha}\right)+2 g \sum_{A} \zeta_{A} \int \mathrm{d}^{2} \theta \mathrm{d}^{2} \bar{\theta} V^{A} \\
& +\int \mathrm{d}^{2} \theta \mathrm{d}^{2} \bar{\theta} \bar{\Phi} \mathrm{e}^{2 g V} \Phi+\int \mathrm{d}^{2} \theta \mathcal{W}(\Phi)+\int \mathrm{d}^{2} \bar{\theta} \overline{\mathcal{W}}(\bar{\Phi}) \\
& =\operatorname{Tr}\left[-\frac{1}{4} F_{\mu \nu} F^{\mu \nu}-\mathrm{i} \lambda \sigma^{\mu} D_{\mu} \bar{\lambda}+\frac{1}{2} D^{2}\right]+\frac{\theta \mathrm{YM}}{32 \pi^{2}} g^{2} \operatorname{Tr} F_{\mu \nu} \tilde{F}^{\mu \nu} \\
& +g \sum_{A} \zeta_{A} D^{A}+\bar{D}_{\mu} \bar{\phi} D^{\mu} \phi-\mathrm{i} \psi \sigma^{\mu} D_{\mu} \bar{\psi}+\bar{F} F+\mathrm{i} \sqrt{2} g \bar{\phi} \lambda \psi \\
& -\mathrm{i} \sqrt{2} g \bar{\psi} \bar{\lambda} \phi+g \bar{\phi} D \phi-\frac{\delta \mathcal{W}}{\delta \phi^{i}} F^{i}-\frac{\delta \overline{\mathcal{W}}}{\delta \bar{\phi}_{i}} \bar{F}_{i}-\frac{1}{2} \frac{\delta^{2} \mathcal{W}}{\delta \phi^{i} \delta \phi^{j}} \psi^{i} \psi^{j}-\frac{1}{2} \frac{\delta^{2} \overline{\mathcal{W}}}{\delta \bar{\phi}^{i} \delta \bar{\phi}^{j}} \bar{\psi}^{i} \bar{\psi}^{j},
\end{aligned}
$$

where $D^{a}$ and $F^{i}$ are auxiliary fields satisfying the equations

$$
D^{a}=-g \bar{\phi} T^{a} \phi-g \zeta^{a}, \bar{F}_{i}=\frac{\delta \mathcal{W}}{\delta \phi^{i}}
$$


and $\mathcal{L}_{\mathrm{FI}}$ is the Fayet-Iliopoulos term.

As a final comment of this Appendix, the information about the group symmetry generators $T^{a}$ will be contained in the kinetic terms of the fields, or in the term $\bar{\Phi} \mathrm{e}^{2 g V} \Phi$.

\section{Appendix D: EWMKE's model: nonlocal $\lambda \phi^{4}$ theory}

In this Appendix, we briefly review the EWMKE (i.e., Eliezer, Woodard, Moffat, Kleppe, Evens) model of a nonlocal and nonsupersymmetric $\lambda \phi^{4}$ theory. Within this framework, the action functional can be split as

$$
\mathcal{S}[\phi]=\mathcal{F}[\phi]+\mathcal{I}[\phi]
$$

where $\mathcal{F}[\phi]$ is the free part, while $\mathcal{I}[\phi]$ is the interaction part. $\mathcal{I}[\phi]$ is supposed to be analytic around the vacuum, and $\mathcal{F}$ takes the form

$$
\mathcal{F}[\phi]=\frac{1}{2} \int \mathrm{d}^{D} x \phi_{i} F_{i j} \phi_{j}
$$

The action $S$ acquires nonlocal nature through a smearing operator $\mathcal{E}$. The EWMKE choice is

$$
\mathcal{E}=\exp \left[\frac{\mathrm{F}}{2 \Lambda^{2}}\right]
$$

whit $\Lambda$ a cutoff scale of new physics beyond effective quantum field theories. The fields $\phi$ are smeared according to

$$
\hat{\phi}_{i}=\mathcal{E}_{i j}^{-1} \phi_{j}
$$

We also define the operator

$$
\mathcal{O} \equiv\left(\mathcal{E}^{2}-I\right) F^{-1}
$$

At this stage, we introduce an auxiliary field $\varphi_{i}$ for each matter field $\phi_{i}$ in the form

$$
\begin{gathered}
\mathcal{S}[\phi, \varphi]=\mathcal{F}[\hat{\phi}]-\mathcal{A}[\varphi]+\mathcal{I}[\phi+\varphi], \\
\mathcal{A}[\varphi]=\frac{1}{2} \int \mathrm{d}^{D} x \varphi_{i} \mathcal{O}_{i j} \varphi_{j} .
\end{gathered}
$$

The classical shadow field equation is

$$
\frac{\delta \mathcal{S}[\phi, \varphi]}{\delta \varphi(x)}=0 .
$$

The nonlocal action is obtained by substituting the solution of the classical field equation,

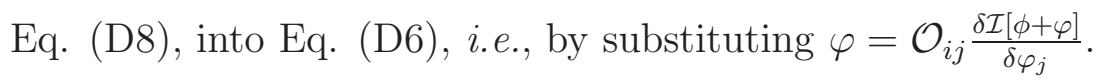




\section{Appendix E: Quantization in the EWMKE model}

In this section, we briefly review quantization issues, as discussed in Refs. [26 30]. Consider therefore the vacuum expectation value of an arbitrary operator $\mathcal{O}$ as

$$
<\mathcal{T}\{\mathcal{O}[\phi]\}>_{\mathcal{E}}=\int \mathcal{D} \phi \mu[\phi](G . F .) \mathcal{O}[\hat{\phi}] \exp \{\hat{\mathrm{iS}}[\phi]\}
$$

where $\mathcal{T}$ is the time-ordering operator, and G.F. is the gauge fixing. $\mathcal{O}$ is nonlocally regulated in a nonlocal theory. Eq. (E1) defines the quantization. The problem of a consistent quantization of a nonlocal field theory is reduced to the problem of existence of the measure factor $\mu[\phi]$ and the gauge fixing. These are necessary in order to preserve unitarity.

The perturbative unitarity of a nonlocal quantum field theory was discussed in papers cited above, applying the Cutkosky rules to EWMKE nonlocal theories where poles are only the zeros of $F=0$, and where interaction vertices are functions of $F$ only. Under these quite general requirements, unitarity on a large subspace of states $\mathcal{M}$, in the Fock space, was shown. $\mathcal{M}$ can also contain ghost fields of BRST quantization, as unphysical polarizations. Gauge invariance guarantees the ghosts' decoupling and unitarity. In the nonlocal procedure, we start from a local quantum field theory, smearing fields and delocalizing vertices. If a continuos transformation $\delta \phi_{i}^{1}=T_{i}\left[\phi^{1}\right]$ generates a symmetry of the local action $S\left[\phi^{1}\right]$, the corresponding transformation of the nonlocal one is $\hat{\delta} \phi_{i}^{1}=\mathcal{E}_{i j}^{2} T_{j}\left[\phi^{1}+\phi^{2}\left[\phi^{1}\right]\right]$, as mentioned above. Such a symmetry has to preserve $\mathcal{D} \phi \mu[\phi]$ of the nonlocal quantum field theory. This corresponds to the following relation:

$$
\hat{\delta}[\log (\mu[\phi])]=-\operatorname{Tr}\left[\frac{\delta \hat{\delta} \phi_{i}}{\delta \phi_{j}}\right]=-\operatorname{Tr}\left[\mathcal{E}_{i k}^{2} \frac{\delta T_{k}}{\delta \phi_{l}}\left[\phi_{1}+\phi_{2}\left[\phi_{1}\right]\right] K_{l k}\left[\phi_{1}+\phi_{2}\left[\phi_{1}\right]\right] \mathcal{O}_{k j}^{-1}\right] .
$$

Under this quantization procedure, we can recover Feynman rules of $\hat{S}\left[\phi_{1}, \phi_{2}\right]$ as simple extension of usual ones: propagators are smeared by a factor $\mathcal{E}^{2}$, and vertices remains the same, as mentioned above. The $\phi_{2}$ are auxiliary fields propagating only off-shell, because they are projected-out by solutions of classical field equations $\phi_{2}\left[\phi_{1}\right]$. All details are extensively discussed in papers just cited above in this same appendix.

[1] S. Tomonaga, Prog. Theor. Phys. 1, 27 (1946).

[2] J. Schwinger, Phys. Rev. 73, 416 (1948). 
[3] J. Schwinger, Phys. Rev. 74, 1439 (1948).

[4] R. P. Feynman, Phys. Rev. 76, 769 (1949).

[5] R. P. Feynman, Phys. Rev. 76, 749 (1949).

[6] R. P. Feynman, Phys. Rev. 80, 440 (1950).

[7] P. A. M. Dirac, in Scientific American (May 1963), p. 53.

[8] M. B. Green, J. H. Schwarz, and E. Witten, Superstring Theory (Cambridge University Press, Cambridge, 1987); J. Polchinsky, String Theory (Cambridge University Press, Cambridge, 1998).

[9] C. Rovelli, Quantum Gravity (Cambridge University Press, Cambridge, 2004).

[10] F. Lizzi, arXiv:0811.0268 [hep-th].

[11] A. Sagnotti, J. Phys. A 46, 214006 (2013).

[12] A. Addazi and M. Bianchi, JHEP 1412, 089 (2014).

[13] A. Addazi, arXiv:1501.04660 [hep-ph].

[14] A. Addazi and M. Bianchi, arXiv:1502.01531 [hep-ph].

[15] A. Addazi and M. Bianchi, arXiv:1502.08041 [hep-ph].

[16] N. Arkani-Hamed, S. Dimopoulos, G. Dvali and G. Gabadadze, hep-th/0209227.

[17] G. Cusin, J. Fumagalli, M. Maggiore, JHEP 1409, 181 (2014).

[18] A. Conroy, T. Koivisto, A. Mazumdar and A. Teimouri, Class. Quantum Grav. 32, 015024 (2015).

[19] N. Arkani-Hamed, H. Georgi and M. D. Schwartz, Annals Phys. 305 (2003) 96.

[20] V. A. Rubakov, arXiv:hep-th/0407104.

[21] S. L. Dubovsky, JHEP 0410, 076 (2004).

[22] A. Addazi and S. Capozziello, arXiv:1407.4840 [gr-qc].

[23] G. Wataghin, Z. Phys. 88, 92 (1934).

[24] D. Kirzhnits, A. Uspekhi Fiz. Nauk. 90, 129 (1966).

[25] G. V. Efimov, Commun. Math. Phys. 5, 42 (1967).

[26] D. A. Eliezer and R. P. Woodard, Phys. Rev. D 40, 465 (1989).

[27] D. A. Eliezer and R. P. Woodard, Nucl. Phys. B 325, 389 (1989).

[28] J. W. Moffat, Phys. Rev. D 41, 1177 (1990).

[29] G. Kleppe and R. P. Woodard, Nucl. Phys. B 388, 81 (1992).

[30] D. Evens, J. W. Moffat, G. Kleppe, and R. P. Woodard, Phys. Rev. D 43, 499 (1991). 
[31] A. Jain and S. D. Joglekar, Int. J. Mod. Phys. A 19, 3409 (2004).

[32] S. D. Joglekar, hep-th/0601006.

[33] M. T. Grisaru, W. Siegel, and M. Rocek, Nucl. Phys. B 159, 429 (1979).

[34] N. Seiberg, Phys. Lett. B 318, 469 (1993).

[35] S. Talaganis, T. Biswas and A. Mazumdar, arXiv:1412.3467 [hep-th].

[36] T. Biswas, E. Gerwick, T. Koivisto and A. Mazumdar, Phys. Rev. Lett. 108, 031101 (2012).

[37] T. Biswas, A. Mazumdar and W. Siegel, JCAP 0603, 009 (2006).

[38] T. Biswas and N. Okada, arXiv:1407.3331 [hep-ph].

[39] X. O. Camanho, J. D. Edelstein, J. Maldacena, and A. Zhiboedov, arXiv:1407.5597 [hep-th].

[40] G. D'Appollonio, P. Di Vecchia, R. Russo and G. Veneziano, arXiv:1502.01254 [hep-th].

[41] M. Bianchi and P. Teresi, JHEP 1201, 161 (2012).

[42] M. Bianchi and A. V. Santini, JHEP 0612, 010 (2006).

[43] J. Wess and J. Bagger, Supersymmetry and Supergravity (Princeton University Press, Princeton, 1992).

[44] H. Widom, Bull. Sci. Math. 104, 19 (1980).

[45] P. B. Gilkey, Invariance Theory, The Heat Equation, and The Atiyah-Singer Index Theorem (CRC Press, Boca Raton, 1995).

[46] B. Booss-Bavnbek and D. Bleecker, Index Theory and Its Applications to Mathematics and Physics (International Press, Boston, 2013).

[47] S. Weinberg, The Quantum Theory of Fields, Volume 1: Foundations (Cambridge University press, Cambridge, 2005) Chapter 4.

[48] N. Bogoliubov and D. V. Shirkov, Introduction to The Theory of Quantized Fields (John Wiley, New York, 1980) pp. 200-220.

[49] K. Fujikawa and W. Lang, Nucl. Phys. B 88, 61 (1975).

[50] B. Ovrut and J. Wess, Phys. Rev. D 25, 409 (1982).

[51] S. Ferrara and P. Piquet, Nucl.Phys. B 93, 261 (1975).

[52] A. Haque and S. D. Joglekar, J. Phys. A 42, 065401 (2009).

[53] Y. Kobayashi and S. Sasaki, Phys. Rev. D 72, 065015 (2005).

[54] S. W. Hawking, in Quantum Gravity 2, a Second Oxford Symposium, edited by C. J. Isham, R. Penrose, and D. W. Sciama (Oxford University Press, Oxford, 1981).

[55] B. S. DeWitt, in 50 Years of Yang-Mills theory, edited by G. 't Hooft (World Scientific, 
Singapore, 2005).

[56] Y. Foures-Bruhat, Acta Math. 88, 141 (1952). 